\title{
Perfect fluid tori orbiting Kehagias-Sfetsos naked singularities
}

\author{
Z. Stuchlík ${ }^{\mathrm{a}}$, D. Pugliese ${ }^{\mathrm{b}}$, J. Schee ${ }^{\mathrm{c}}$, H. Kučákovád \\ Faculty of Philosophy and Science, Institute of Physics and Research Centre of Theoretical Physics and Astrophysics, Silesian University in Opava, \\ Bezručovo náměstí 13, 74601 Opava, Czech Republic
}

Received: 11 May 2015 / Accepted: 6 September 2015 / Published online: 25 September 2015

(c) The Author(s) 2015. This article is published with open access at Springerlink.com

\begin{abstract}
We construct perfect fluid tori in the field of the Kehagias-Sfetsos (K-S) naked singularities. These are spherically symmetric vacuum solutions of the modified Hořava quantum gravity, characterized by a dimensionless parameter $\omega M^{2}$, combining the gravitational mass parameter $M$ of the spacetime with the Hořava parameter $\omega$, reflecting the role of the quantum corrections. In dependence on the value of $\omega M^{2}$, the $\mathrm{K}-\mathrm{S}$ naked singularities demonstrate a variety of qualitatively different behavior of their circular geodesics that is fully reflected in the properties of the toroidal structures, demonstrating clear distinction to the properties of the torii in the Schwarzschild spacetimes. In all of the K-S naked singularity spacetimes the tori are located above an "antigravity" sphere where matter can stay in a stable equilibrium position, which is relevant for the stability of the orbiting fluid toroidal accretion structures. The signature of the K-S naked singularity is given by the properties of marginally stable tori orbiting with the uniform distribution of the specific angular momentum of the fluid, $l=$ const. In the $\mathrm{K}-\mathrm{S}$ naked singularity spacetimes with $\omega M^{2}>0.2811$, doubled tori with the same $l=$ const can exist; mass transfer between the outer torus and the inner one is possible under appropriate conditions, while only outflow to the outer space is allowed in complementary conditions. In the $\mathrm{K}-\mathrm{S}$ spacetimes with $\omega M^{2}<0.2811$, accretion from cusped perfect fluid tori is not possible due to the non-existence of unstable circular geodesics.
\end{abstract}

\section{Introduction}

The Hořava quantum gravity [1] is a recent promising approach to the quantum gravity that has attracted much

\footnotetext{
a e-mail: zdenek.stuchlik@physics.cz

be-mail: daniela.pugliese@fpf.slu.cz

ce-mail: jan.schee@fpf.slu.cz

d e-mail: hana.kucakova@centrum.cz
}

attention, being a field-theory based on the ideas of solid state physics that use an anisotropic scaling of space and time [2]. The time dimension is scaled as $t \rightarrow b^{z} t$, with an integer $z$, while the space dimensions are scaled as $x \rightarrow b x$ and, as a consequence, its Lagrangian is Lorentz invariant at low energies, but the Lorentz invariance is broken at high energies. In fact, the parameter $z$ changes from $z=3$, in the ultraviolet (UV) limit, to $z=1$ in the infrared (IR) limit where the Lorentz invariance is recovered [1,310]. The solutions of the Hořava-Lifshitz effective gravitational equations have been discovered in [11-13], while a spherically symmetric solution of the modified Hořava gravity, compatible with the Minkowski vacuum and having a asymptotically Schwarzschildian character is known as the Kehagias-Sfetsos (K-S) metric [14,15]. The K-S solution is a special class of solutions found by Kiritsis and Kofinas [16].

The character of the $\mathrm{K}-\mathrm{S}$ geometries is governed by the dimensionless product $\omega M^{2}$ where the parameter $M$ is the gravitational mass determining the distance scales in the $\mathrm{K}-$ $\mathrm{S}$ metric, and $\omega$ is the Horava parameter, which reflects the influence of the quantum effects. ${ }^{1}$ If we assume that the Hořava parameter is a universal constant, then the character of the spacetime is only regulated by the mass $M$ : for $\omega M^{2} \geq 1 / 2$, the $\mathrm{K}-\mathrm{S}$ metric describes a black hole solution, while for $\omega M^{2}<1 / 2$, there are no event horizons and the metric describes a naked singularity. The current observa-

\footnotetext{
1 The Horrava parameter $\omega$ is an universal constant for the spherically symmetric, asymptotically flat solution. To be more specific it is a parameter directly related to the Lagrangian parameters as we have $\omega=8 \mu^{2}(3 \lambda-1) / \kappa^{2}$ for the spherically symmetric solutions. The value $\lambda=1$ corresponds to an IR fixed point where the standard general relativity is recovered, and the solution is asymptotically flat, the case $\omega=16 \mu^{2} / \kappa^{2}$. The parameters $\{\lambda, \kappa, \mu\}$ enter the action for the quantum gravity with the following meaning: the coupling parameter $\lambda$ regulating the contribution of the trace of the extrinsic curvature, $\kappa$ is a dimensionless coupling constant, whereas $\mu$ has mass dimension $[\mu]=1$. A deeper and detailed discussion of the origin of the $\omega$ parameter can be found in [14].
} 
tional limits on $\omega$ [17-19] do not exclude the existence of stellar-mass $\mathrm{K}-\mathrm{S}$ naked singularities. In fact, various tests indicate values compatible with the naked singularity, not a black hole solution for the family of $\mathrm{K}-\mathrm{S}$ metrics. There is therefore great interest in a deeper analysis of the class of naked singularities. Specifically a solar system test puts the limit $\omega>3.2 \times 10^{-20} \mathrm{~cm}^{-2}$ [19], implying that the gravitational mass of $\mathrm{K}-\mathrm{S}$ naked singularities cannot exceed $2.6 \times 10^{4} M_{\odot}$. Thus, it is of key interest to search for any signatures of $\mathrm{K}-\mathrm{S}$ naked singularity spacetimes, for example in the emergence of accretion phenomena, which we are allowed to distinguish clearly from the black hole attractors. Therefore there is an obvious interest in studying the $\mathrm{K}-\mathrm{S}$ naked singularity spacetimes. The $\mathrm{K}-\mathrm{S}$ black hole spacetimes have been investigated in a series of works on the motion of particles [19-25] and optical phenomena [26-29]. Then, as in [30], one could study these objects by analyzing the power spectrum of a simple accretion disk model, a similar analysis should show traces of the presence of the free horizon singularity for geometries with similar dynamical properties of orbiting matter.

The $\mathrm{K}-\mathrm{S}$ naked singularity spacetimes were recently extensively studied, in particular the effective potential of the test particle motion and the circular geodesics were studied in [31], where a close similarity with properties of the circular geodesics of the Reissner-Nordström (RN) naked singularity spacetimes [32,33] has been demonstrated. The ultra-high-energy particle collisions in the deepest parts of the gravitational potential of $\mathrm{K}-\mathrm{S}$ naked singularity spacetimes have been studied in [34]. A wide range of optical phenomena related to the innermost parts of Keplerian discs (direct and indirect images, spectral continuum of thermal radiation, and profiled spectral lines) had been exposed in [35]. For all of the considered phenomena related to the $\mathrm{K}-\mathrm{S}$ naked singularities, an "antigravity" effect [31,35] plays a fundamental role; a similar situation has also been discovered in braneworld naked singularity spacetimes [36-40]. However, it seems to be different in comparison to the rotating Kerr naked singularity spacetimes [41-48]; for a detailed analysis of the magnetized thick discs see [49].

In all the aforementioned studies, the motion of photons, particles, and fluids has been assumed to be governed by the geodetic structure of the spacetime, i.e. by the IR (low energy) end of the Hořava theory, and possible effects of the Lorentz invariance violation, expected at the UV (high energy) end of the theory were not considered [1,3, 14, 15]. Thus, the Lorentz invariance violation remains still an open issue of the Hořava gravity, although the extremely complex character of the particle motion in a situation where this violation is relevant has been exposed in [50]. Here we keep the assumption of the standard geodesic motion used in the previous research.
All the K-S naked singularity spacetimes contain a "static" sphere (or an "antigravity" sphere): the test particles, subject to an "antigravity" effect, are in a stable equilibrium position. This sphere radius also determines the location of the innermost limit on the existence of circular geodesics, corresponding to orbits with zero angular momentum and zero angular frequency $[31,35]$. The properties of the circular geodesics in the $\mathrm{K}-\mathrm{S}$ naked singularity spacetimes are strongly determined by the dimensionless parameter $\omega M^{2}$ [31,34,35]: as $0<\omega M^{2}<\left(\omega M^{2}\right)_{\text {mso }}=0.2811$, stable circular orbits exist between the static sphere at radius $r_{\text {stat }}$ and infinity, while the spacetimes $\left(\omega M^{2}\right)_{\text {mso }}<\omega M^{2}<$ $\left(\omega M^{2}\right)_{\gamma}=0.3849$ are characterized by two separated regions of stable circular geodesics. The outer region extends between the inner marginally stable orbit at $r_{i s c o}$ and infinity and the inner one extends between the static radius $r_{\text {stat }}$ and the outer marginally stable orbit at $r_{\text {osco }}$, unstable circular geodesics are located at $r_{\text {osco }}<r<r_{i s c o}$. In the $\mathrm{K}-\mathrm{S}$ spacetime, with $\left(\omega M^{2}\right)_{\gamma}<\omega M^{2}<1 / 2$, the inner region of stable circular orbits is bounded by a (stable) photon circular orbit located $r_{\gamma}^{o}$, while the unstable circular geodesics, located at $r_{\gamma}^{i}<r<r_{i s c o}$, are bounded by the (unstable) photon circular orbit in $r_{\gamma}^{i}$, located under the outer region of stable circular geodesics: then no circular geodesics are possible in the region $r_{\gamma}^{o}<r<r_{\gamma}^{i}$ [31].

The standard Keplerian accretion disc can exist in the region of the outer stable circular geodesics, which is the region of the stable circular geodesics in the $\mathrm{K}-\mathrm{S}$ geometries with $0<\omega M^{2}<\left(\omega M^{2}\right)_{\text {mso }}=0.2811$, while in the other K-S naked singularity spacetimes, the Keplerian disc has to be limited by $r_{i s c o}$ from below [35]. The gradient of the angular frequency $\Omega_{K}$ of the circular geodesic motion vanishes at some radius $r_{\Omega}^{\mathrm{Max}}$ inside the Keplerian disc in all the $\mathrm{K}-\mathrm{S}$ naked singularity spacetimes, and the condition for Keplerian accretion, $\mathrm{d} \Omega_{K} / \mathrm{d} r<0$, is then violated [35]. We expect that near the radius $r_{\Omega}^{\mathrm{Max}}$, where the Keplerian accretion stops to work, matter has to be accumulated and a fluid toroidal structure has to be created successively. Therefore, in the $\mathrm{K}-\mathrm{S}$ naked singularity spacetimes the fluid toroidal structures have to be related even to accretion processes that were of the Keplerian character at the beginning. Of course, the toroidal perfect fluid equilibrium configurations are related also to the inner regions of the circular geodesics, and could thus be doubled, as in the case of the RN naked singularity spacetimes [51].

Here, we study the structure and shape of perfect fluid toroidal equilibrium tori orbiting the $\mathrm{K}-\mathrm{S}$ naked singularities. We consider the tori for the whole range of the dimensionless parameter $\omega M^{2}<1 / 2$ corresponding to naked singularity spacetimes. All the various kinds circular geodesic structures will be considered and their impact on the structure of the toroidal configurations will be reflected. 
The present article is structured as follows: in Sect. 2 we introduce the Kehagias-Sfetsos $(\mathrm{K}-\mathrm{S})$ geometry. Section 3 is devoted to the analysis of circular geodesics in KehagiasSfetsos spacetimes. In Sect. 4 we discuss the structure of toroidal perfect fluid orbiting a Kehagias-Sfetsos attractor, and the equilibrium tori in the $\mathrm{K}-\mathrm{S}$ naked singularity spacetimes are considered in Sect. 5. A classification of toroidal configurations in $\mathrm{K}-\mathrm{S}$ spacetimes is introduced and detailed in Sect. 6. Finally the article draws conclusions in Sect. 7.

\section{Kehagias-Sfetsos geometry}

The Kehagias-Sfetsos solution of the modified Hořava gravity in the standard Schwarzschild coordinates and the geometric units ( $c=G=1)$ reads [14]

$$
\begin{aligned}
& \mathrm{d} s^{2}=-f(r) \mathrm{d} t^{2}+\frac{1}{f(r)} \mathrm{d} r^{2}+r^{2} \mathrm{~d} \theta^{2}+r^{2} \sin ^{2} \theta \mathrm{d} \phi^{2}, \\
& f(r) \equiv 1+r^{2} \omega\left(1-\sqrt{1+4 M / \omega r^{3}}\right),
\end{aligned}
$$

where $\omega$ is a free parameter governing the role of the modified Hořava quantum gravity, the parameter $M$ gives the gravitational mass and the dimensional scale of the solution. Although the $\mathrm{K}-\mathrm{S}$ solution of the modified Hořava gravity is a vacuum solution, it is not a Ricci-flat solution. In the Schwarzschild limit, $\omega \rightarrow \infty$, the Ricci scalar has the asymptotic behavior $R \sim 1 / \omega$. More information can be found $^{2}$ in [34]. The physical singularity of the K-S spacetimes located at $r=0$ is of a special character, as the metric coefficients $g_{t t}(r=0)=g_{r r}(r=0)=1$ are finite there, while their radial derivatives are divergent. This implies that particles freely radially falling in from infinity can reach the physical singularity, if they do not lose energy during their fall. However, any particle with non-zero angular momentum is repulsed by the centrifugal repulsive barrier, acting in accord with the "antigravity" effect, and cannot reach the physical singularity [31]. The "antigravity" effect can be illustrated by the behavior of the lapse function $f(r)$ in the vicinity of the physical singularity. For $r \rightarrow 0$, we have

\footnotetext{
${ }^{2}$ We stress that this work covers the infrared limit of the modified Hořava quantum gravity, and particularly the Kehagias-Sfetsos class of asymptotically flat metrics. The physical context is therefore completely different from the Einstein gravity: these non-zero terms are not associated to matter fields of any sort, but they have to be considered a purely quantum effect. In the IR regime we consider here, it is the only significant remnant of the quantum nature of the spacetime. Therefore, it has a purely (quantum) geometric origin, no attempt to interpret it as a consequence of some matter contribution is proposed here. To be more specific, the nonzero terms, the Ricci terms, are a consequence of the quantum nature of the geometry and particularly of the anisotropy between the space and time properly of the Hořava gravity at the quantum level, encoded in the $\omega$ parameter. It is not induced by any deformation of some vacuum solutions of the Einstein equations.
}

$f(r) \sim 1-2 \sqrt{\omega M r}$, indicating gravitation repulsion of the quintessential-field type [52].

The properties of the $\mathrm{K}-\mathrm{S}$ spacetimes are governed by the dimensionless parameter $\omega M^{2}[31,34,35]$. If the Hořava parameter $\omega$ is assumed fixed, then the gravitational mass parameter $M$ governs the character of the $\mathrm{K}-\mathrm{S}$ spacetimes. Two horizons of the $\mathrm{K}-\mathrm{S}$ black hole spacetimes exist at the radii

$r_{ \pm} \equiv M \pm \sqrt{M^{2}-\frac{1}{2 \omega}}$ if $\omega M^{2} \geq \omega M_{h}^{2} \equiv \frac{1}{2}$.

When equality holds, the horizons coincide, giving an extreme black hole (BH) K-S spacetime. The $\mathrm{K}-\mathrm{S}$ naked singularity (NS) spacetimes occur for $\omega M^{2}<\omega M_{h}^{2}$. Therefore, assuming the parameter $\omega$ fixed, the gravitational mass $M$ being small enough guarantees the existence of a $\mathrm{K}-\mathrm{S}$ naked singularity. ${ }^{3}$ Recent work on the influence of the dimensionless Hořava parameter in astrophysical systems $[26,53,54]$ set the very weak limit $\omega M^{2}>8 \times 10^{-10}$, which is very far from the limit corresponding to the $\mathrm{K}-\mathrm{S}$ black hole spacetimes. K-S naked singularity spacetimes have to be considered in astrophysical phenomena related to compact objects having mass $M<10^{4} M_{\odot}$, if the modified Hořava gravity is considered relevant.

\section{Circular geodesics}

The motion of test particles and photons is governed by the spacetime geodesics. Due to the axial symmetry and stationarity of the $\mathrm{K}-\mathrm{S}$ spacetimes two constants of the geodesic motion arise being related to the covariant components of the particle (photon) 4-momentum $P_{\phi}=L, P_{t}=-E$. The radial component of the 4-momentum of test particles reads

$\left[P^{r}\right]^{2}=E^{2}-V_{\mathrm{eff}}, \quad V_{\mathrm{eff}} \equiv f(r)\left(m^{2}+\frac{L^{2}}{r^{2}}\right)$,

where an effective potential $V_{\text {eff }}$ is introduced, $m$ being the particle mass. Circular geodesics were studied for the $\mathrm{K}-\mathrm{S}$ black hole spacetimes in $[22,55]$ and for the K-S naked singularity spacetimes in $[31,34,35]$. We give a short summary of the properties of the circular geodesics in dependence on the parameter $\omega M^{2}$.

\footnotetext{
3 As the K-S metrics are stationary one could even consider the Komar mass. However, this definition will contain terms related to the Ricci tensor and the $\omega$ parameter characterizing this non-Ricci-flat solution. An attempt to characterize the mass definition as well as the thermodynamical quantities in the $\mathrm{K}-\mathrm{S}$ geometries can be found in [69-75].
} 


\subsection{Photon circular geodesics}

We consider the equatorial motion $(\theta=\pi / 2)$; the photon orbits exist for

$\omega M^{2}>\omega M_{\gamma}^{2} \equiv \frac{2}{3 \sqrt{3}}$.

In $\mathrm{K}-\mathrm{S}$ black hole spacetimes, $\omega M^{2} \geq \omega M_{h}^{2}=1 / 2$, one photon circular geodesic always exists, and its radius is given by

$r_{\gamma}^{i} \equiv 2 \sqrt{3} M \cos \left(\frac{1}{3} \cos ^{-1}\left(-\frac{2}{3 \sqrt{3} \omega M^{2}}\right)-\frac{2 \pi}{3}\right)$.

This orbit is unstable relative to radial perturbations and represents an inner boundary for the existence of circular geodesics. For the $\mathrm{K}-\mathrm{S}$ naked singularity spacetimes with $\omega M_{h}^{2}>\omega M^{2}>\omega M_{\gamma}^{2}$, two photon circular orbits exist - the outer (unstable) one at the radius given by Eq. (6) and the inner (stable) one at

$r_{\gamma}^{o} \equiv 2 \sqrt{3} M \cos \left(\frac{1}{3} \cos ^{-1}\left(-\frac{2}{3 \sqrt{3} \omega M^{2}}\right)\right)$.

Between the stable and unstable circular orbits no circular geodesics are possible. This stable circular photon orbit is the outer boundary of the inner region of the circular geodetic orbits. As $r_{\gamma}^{o}(\omega)<r_{\gamma}^{i}(\omega)$, hereafter we adopt the notation $r_{\gamma}^{-} \equiv r_{\gamma}^{o}$ and $r_{\gamma}^{+} \equiv r_{\gamma}^{i}$, so that $r_{\gamma}^{-}<r_{\gamma}^{+}$. For $\omega M^{2}=$ $\omega M_{\gamma}^{2}$, only one photon circular orbit at the radius $r_{\gamma}=\sqrt{3} M$ remains, while for $\omega M^{2}<\omega M_{\gamma}^{2}$, no photon circular orbit exists.

In the $\mathrm{K}-\mathrm{S}$ naked singularity spacetimes with $\omega M_{h}^{2}>$ $\omega M^{2}>\omega M_{\gamma}^{2}$, allowing for the existence of the stable and unstable photon circular geodesics, a region of trapped photon orbits exists in the vicinity of the stable photon circular geodesic. This situation is similar to those discovered in the field of Kerr naked singularity spacetimes [42], where the trapped photons can strongly influence the accretion phenomena, especially in the near-extreme Kerr naked singularity states $[41,43,45,56]$. We expect a similar strong influence in the $\mathrm{K}-\mathrm{S}$ naked singularity spacetimes $[31,35]$. In the following, we simplify the calculations, putting $M=1$ and making both the radial coordinate and the Hořava parameter dimensionless; of course, also the time coordinate becomes dimensionless. The resulting formulas can easily be transformed to expressions containing the mass parameter $M$ by the transformations $r / M \rightarrow r$ and $\omega M^{2} \rightarrow \omega$.

\subsection{Keplerian circular orbits}

The equatorial circular orbits of test particles are determined by the condition $\mathrm{d} V_{\mathrm{eff}} / \mathrm{d} r=0$. The radial profile of the specific angular momentum $L_{K}$ (related to the unit rest mass of the particle) and energy $E_{K}$ take the form

$$
\begin{aligned}
\frac{L_{K}^{2}(r ; \omega)}{m^{2}} \equiv & \frac{r^{2}}{r A(r ; \omega)-3}\left[r^{3} \omega A(r ; \omega)-\left(1+r^{3} \omega\right)\right], \\
A(r ; \omega) \equiv & \sqrt{1+\frac{4}{r^{3} \omega}} \\
\frac{E_{K}^{2}(r ; \omega)}{m^{2}} \equiv & {\left[1+r^{2} \omega\left(1-\sqrt{1+\frac{4}{r^{3} \omega}}\right)\right] } \\
& \times\left(1+\frac{1}{r A-3}\left[r^{3} \omega A-\left(1+r^{3} \omega\right)\right]\right) .
\end{aligned}
$$

In the following, we use the simplified notation $L_{K} / m \rightarrow$ $L_{K}$ and $E_{K} / m \rightarrow E_{K}$. The radial profile of the angular frequency of the test particle motion on the Keplerian (geodesic) circular orbits takes the form

$\Omega_{K}(r ; \omega) \equiv \frac{U^{\phi}}{U^{t}}=\frac{f(r)}{r^{2}} \frac{L_{K}}{E_{K}}=\sqrt{\frac{r^{3} \omega[A(r ; \omega)-1]-1}{r^{3} A(r ; \omega)}}$.

The radial profile of the specific angular momentum demonstrates a zero point $\left(L_{K}^{2}(r ; \omega)=0\right)$ giving the static, or "antigravity", radius where a stable equilibrium position of the test particles occurs giving the lower limit for the existence of circular orbits of test particles in the field of all K-S naked singularity spacetimes. The static radius is given by [31]

$r_{\text {stat }}(\omega) \equiv \frac{1}{(2 \omega)^{1 / 3}}$

No zero points of the specific energy radial profile exist. At the static radius, the specific energy of the Keplerian circular orbits reaches its minimum $E_{K}\left(r=r_{\text {stat }}, \omega\right) \equiv E_{\text {stat }}=$ $1-(2 \omega)^{1 / 3}$. Since $L_{K}=0$ at the static radius, there is naturally also $\Omega_{K}\left(r_{\text {stat }}, \omega\right)=0$ in all K-S naked singularity spacetimes. This implies that a turning point (maximum) of the radial profile of the Keplerian angular velocity has to exist. The condition $\mathrm{d} \Omega_{K} / \mathrm{d} r=0$ implies the location of the local maximum to be at

$r_{\Omega}^{\operatorname{Max}}(\omega) \equiv\left(\frac{2}{\omega}\right)^{1 / 3}=4^{1 / 3} r_{\text {stat }}(\omega)$

i.e., close to the static radius. The function $r_{\Omega}^{\operatorname{Max}}(\omega)$ terminates at $r_{\Omega}^{\operatorname{Max}}\left(\omega=\omega_{\gamma}\right)=\sqrt{3}=r_{\gamma}\left(\omega=\omega_{\gamma}\right)$. The circular geodesics with zero gradient of the angular velocity exist only in the $\mathrm{K}-\mathrm{S}$ naked singularity spacetimes with $\omega<\omega_{\gamma}$. In the spacetimes with $\omega_{h}>\omega>\omega_{\gamma}$, the radius is located in the region forbidden for the circular geodesics - the angular velocity increases with decreasing radius in the external region of the circular geodesics located above the $r_{\gamma}^{+}$, and it 
decreases with decreasing radius in the internal region of the circular geodesics, located between $r_{\text {stat }}$ and $r_{\gamma}^{-}$- see $[31,35]$. The marginally stable circular geodesics satisfy simultaneously the conditions $\mathrm{d} V_{\text {eff }} / \mathrm{d} r=0$ and $\mathrm{d}^{2} V_{\text {eff }} / \mathrm{d} r^{2}=0$; these lead to the orbital angular momentum

$L_{m s o}^{2}(r ; \omega) \equiv \frac{r^{2}\left[2-4 r^{3} \omega(2-A(r))-r^{6} \omega^{2}(1-A(r))\right]}{30-3 A(r) r\left(4+r^{3} \omega\right)+12 r^{3} \omega}$.

The critical value of the Hořava parameter, $\omega_{m s o}$, separating spacetimes admitting two marginally stable radii $r_{\text {osco }}$ and $r_{i s c o}$ and no marginally stable orbits, reads $\omega_{m s o} \equiv$ 0.281100 ; we have $r_{\text {osco }} \leq r_{i s c o}$. Hereafter we adopt the notation $r_{m s o}^{-} \equiv r_{o s c o}$ and $r_{m s o}^{+} \equiv r_{i s c o}$, so that $r_{m s o}^{-} \leq$ $r_{m s o}^{+}$. The marginally bound orbits, i.e., the unstable circular geodesics having the specific energy $E_{K}=1$ are given by the condition $E_{m b o} \equiv E_{K}\left(r_{m b o}, L_{m b o}\right)=1$, which governs both the radius $r_{m b o}$ and the angular momentum $L_{m b o}$ of the Keplerian orbit. Note that this condition determines both the unstable outer orbits with $E_{K}=1$ and $L_{K}=L_{m b o}$ and the stable inner orbits with the same energy $E_{K}=1$, but different angular momentum $L>L_{m b o}$, if they exist. Clearly, these orbits can exist only in the spacetimes allowing for the existence of two stable circular geodesics for a given angular momentum, i.e. the spacetimes with $\omega>\omega_{m s o}$. There exists a critical value of the Hořava parameter, $\omega_{m b o} \equiv 0.327764$, separating spacetimes where the marginally bound unstable circular orbits are allowed, and where they do not exist, since the inner (outermost) marginally stable orbits have $E_{m s o}^{-}<1$ in such spacetimes. The marginally stable orbits radii $r_{m s o} \in\left(r_{m s o}^{-}, r_{m s o}^{+}\right)$, and the marginally bound orbit radii $r_{m b o}$ are given as functions of the parameter $\omega$ in Fig. 3, along with the radii of the photon circular geodesics $r_{\gamma}\left(r_{\gamma}^{-}, r_{\gamma}^{+}\right)$, the static radius, $r_{\text {stat }}$, and the radius where the gradient of the angular velocity of the circular geodesics vanishes, $r_{\Omega}^{\mathrm{Max}}$. The critical points where the curves $r_{\gamma}(\omega), r_{m b o}(\omega)$, and $r_{m s o}(\omega)$ have the local extremum are determined by the points $C_{i}$ with $i \in\{0, \ldots, 3\}$ of the $(r-\omega)$ plane:

$C_{0} \equiv\left(r_{\gamma}, \omega_{\gamma}\right)=(1.7321,0.3849)$,

$C_{1} \equiv\left(r_{m b o}, \omega_{m b o}\right)=(2.2818,0.327764)$,

$C_{2} \equiv\left(r_{m s o}, \omega_{m s o}\right)=(3.5140,0.2811)$,

$C_{3} \equiv\left(r_{\Omega}^{\mathrm{Max}}, \omega_{m s o}\right)=(1.921,0.2811)$.

For $\omega>1 / 2$ corresponding to the $\mathrm{K}-\mathrm{S}$ black holes, there are no circular orbits under the inner horizon, contrary to the case of the standard general relativistic RN or Kerr black holes. The point $C_{3}$ determining the radius $r_{\Omega}^{\mathrm{Max}}(\omega)$ for the critical value $\omega_{m s o}$ is important for the Keplerian disc structure during the transition across the critical point in the evolution of a $\mathrm{K}-\mathrm{S}$ naked singularity due to the accreting matter. Vanishing of the angular-velocity gradient is relevant for
Keplerian accretion in the K-S spacetimes with $\omega<\omega_{m s o}$, since $r_{\Omega}^{\operatorname{Max}}(\omega)<r_{m s o}(\omega)$ in the spacetimes with $\omega>\omega_{m s o}$. The points $C_{0}$ and $C_{2}$ (or $C_{1}$ ) give the classification of the $\mathrm{K}-\mathrm{S}$ naked singularity spacetimes due to the properties of circular geodesics governing the Keplerian accretion discs. The points $C_{0}, C_{1}$, and $C_{2}$ also give the maximal extension of the inner Keplerian discs in the corresponding classes of K-S naked singularity spacetimes - for details see the discussion in [35]. Here we briefly present the classification of the $\mathrm{K}-\mathrm{S}$ spacetimes reflecting the properties of circular geodesics.

\subsection{On the Keplerian discs and classification of the $\mathrm{K}-\mathrm{S}$ spacetimes}

In dependence on the (dimensionless) parameter $\omega M^{2}$, four classes of K-S spacetimes were introduced in [35]. For each class of the $\mathrm{K}-\mathrm{S}$ spacetimes, typical radial profiles of the specific energy, specific angular momentum, and angular velocity of the circular geodesics governing behavior of the Keplerian discs was presented in Fig. 5 in [35]; the corresponding behavior of the effective potential is reflected in [31]. In all the classes of $\mathrm{K}-\mathrm{S}$ naked singularity spacetimes a sphere of matter can be created at the static radius. Such a sphere could be a final state of the accreted matter if dissipative mechanisms (viscosity, gravitational radiation, etc.) could work at time scales long enough. The four classes of $\mathrm{K}-\mathrm{S}$ spacetimes are given in their dependence on the magnitude of the Hořava dimensionless parameter $\omega$ (using simplification of $M=1$ ).

\subsubsection{Class I: naked singularities with $\omega<\omega_{m s o}$}

The K-S naked singularity spacetimes contain no photon circular orbits and no unstable circular orbits. Only stable circular orbits are allowed for the specific angular momentum from the interval $\left.L^{2} \in\right] 0+\infty$ [, Fig. 1. Since all the circular geodesic orbits are stable, with energy and angular momentum decreasing with decreasing radius, we can consider the Keplerian discs in the whole range of $r \in] r_{\text {stat }},+\infty[$. A critical point of the Keplerian discs in these $\mathrm{K}-\mathrm{S}$ naked singularity spacetimes occurs at the radius $r_{\Omega}^{\mathrm{Max}}$ where the angularvelocity gradient vanishes. At this radius and its vicinity, the standard viscosity mechanism based on the non-zero gradient of the angular velocity of the accreting matter loses its relevance. It can be substituted there by the mechanism based on the gravitational radiation of the orbiting matter, as corresponding losses of energy and angular momentum can transmit the accreting matter to the region where the viscosity mechanism causes heat production and thermally radiating Keplerian discs could continue its work. A more realistic expectation is related to the possibility of cumulation of accreting matter near the radius $r_{\Omega}^{\mathrm{Max}}$ where a fluid 


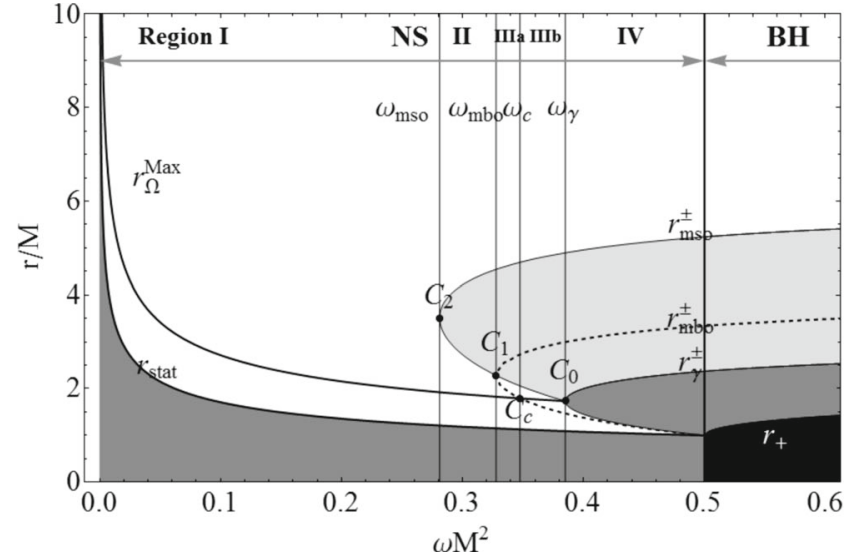

Fig. 1 Left panel regions of the stable and unstable circular geodetical orbits are given in dependence on the Hořava parameter $\omega$ along with the critical radii of the geodesic motion. Naked singularity (NS) case $\left(\omega M^{2} \in\right] 0,0,5[)$ and black hole case (BH) $\left(\omega M^{2}>0,5\right)$. Radii of the set $\mathscr{R} \equiv\left\{r_{m s o}^{ \pm}, r_{m b o}^{ \pm}, r_{\gamma}^{ \pm}, r_{\Omega}^{\text {Max }}, r_{\text {stat }}\right\}$ as a function of the Hořava parameter $\omega$ in the plane $\left(r / M, \omega M^{2}\right)$. The black region is $r<r_{+}$, where $r_{+}$ is the black hole horizon, gray regions are forbidden, no circular motion is possible, regions of unstable circular orbits are in light-gray. White regions mark the stability regions. The marginally bounded orbits are $r_{m b o}^{+}>r_{m b o}^{-}$, and $r_{m s o}^{+}>r_{m s o}^{-}$are marginally stable circular orbits, $r_{\gamma}^{+}>r_{\gamma}^{-}$are limiting circular orbits (photon-like orbits). The radius

toroid with pressure gradients governing its shape and structure could naturally arise. ${ }^{4}$

\subsubsection{Class II: naked singularities with $\omega \in] \omega_{m s o}, \omega_{\gamma}[$}

These $\mathrm{K}-\mathrm{S}$ naked singularity spacetimes containing no photon circular orbits, but two marginally stable circular orbits at $r_{m s o}^{-}$and $r_{m s o}^{+}$Fig. 1. The stable circular orbits, potentially corresponding to Keplerian discs, are located in the region $r \in] r_{\text {stat }}, r_{m s o}^{-}$( (the inner Keplerian disc) and $\left.r \in\right] r_{m s o}^{+}, \infty[$ (the outer Keplerian disc). For the marginally stable circular orbits, the energy and angular momentum satisfy the conditions $E_{m s o}^{+}<E_{m s o}^{-}$and $L_{m s o}^{+}<L_{m s o}^{-}$. The unstable circular geodesics are located at $r \in] r_{m s o}^{-}, r_{m s o}^{+}[$, and their energy and angular momentum belong to the intervals $E \in] E_{m s o}^{+}, E_{m s o}^{-}$[ and $L \in] L_{m s o}^{+}, L_{m s o}^{-}$. Since the critical radius $r_{\Omega}^{\mathrm{Max}}<r_{m s o}^{-}$, we can conclude that the complete outer Keplerian disc is quite regular relative to the MRI viscosity mechanism; the critical point of the vanishing angular-velocity gradient is

\footnotetext{
${ }^{4}$ We can assume that the standard Keplerian accretion discs are limited by the condition $\frac{\mathrm{d} \Omega_{K}}{\mathrm{~d} r}<0$, which is required by the Magneto-Rotational Instability (MRI) viscosity mechanism [57]. If MRI is the only mechanism generating the viscosity effects that enable accretion of matter in the Keplerian discs, their inner edge has to be located at the critical radius $r_{\Omega}^{\mathrm{Max}}$ [31]. If some other viscosity mechanism related to the oppositely oriented gradient of the angular velocity $\left(\mathrm{d} \Omega_{K} / \mathrm{d} r>0\right)$ works, the internal parts of the Keplerian discs could also heat up and radiate thermally.
}

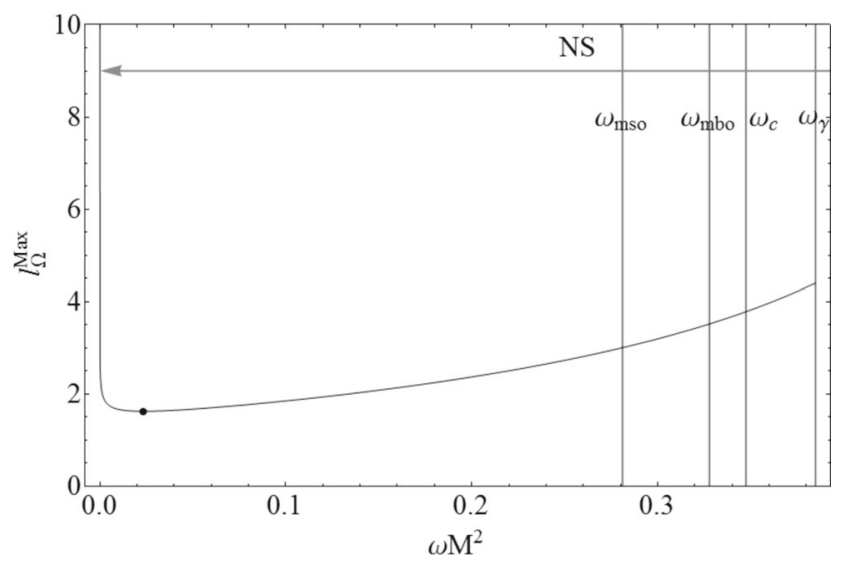

is in units of mass $M$ as $r / M \rightarrow r$ and also $\omega M^{2} \rightarrow \omega$. The static radius $r_{\text {stat }}$ and the radius $r_{\Omega}^{\text {Max }}$ of the local maximum of the angular velocity are also plotted. The standard MRI viscosity mechanism can be at work in the region of stability up to the radius $r_{\Omega}^{\mathrm{Max}}$ where the angular-velocity gradient governing the viscosity vanishes. The critical points $C_{0}, C_{1}, C_{2}, C_{3}$, and $C_{c}$ are defined in the text. Regions $I-I V$ of naked singularity sources are also signed. Right panel the fluid angular momentum $l_{\Omega}^{\mathrm{Max}} \equiv l\left(r_{\Omega}^{\mathrm{Max}}\right)$, evaluated on the critical point $r_{\Omega}^{\mathrm{Max}}$ for the frequency $\Omega$, as a function of the Hořava parameter $\omega$, the point on the curve shows a minimum of $l_{\Omega}^{\mathrm{Max}}$ with respect to $\omega$; see Eq. (27)

related to the inner Keplerian disc only. Therefore, we can conclude that the accretion Keplerian discs work quite well down to the isco circular orbit and the complexities related to the vanishing of the angular-velocity gradient are relevant only in the inner Keplerian discs, if such discs will be created - for details see [35]. In the $\mathrm{K}-\mathrm{S}$ naked singularity spacetimes allowing for the existence of the unstable circular geodesics, the marginally bound (unstable) circular geodesics with specific energy given by the condition $E_{m b o}=1$ play a crucial role. Such orbits can be approached approximately by particles starting at rest at infinity with specific energy $E=1$ and angular momentum $L \sim L_{m b o}$ and are significant in theory of toroidal configurations of perfect fluid orbiting black holes or naked singularities, giving the upper limit on the existence of marginally stable toroidal configurations [5860]. The marginally bound orbits can exist only in the $\mathrm{K}-$ $\mathrm{S}$ naked singularity spacetimes having the Hořava parameter $\omega \in] \omega_{m b o}, \omega_{h}\left[\right.$. In the K-S spacetimes with $\omega<\omega_{b}$ no unstable circular orbits with $E>1$ exist - then even particles following unstable circular orbits can remain on a bounded orbit, if perturbed radially, and the equilibrium toroidal configurations of perfect fluid cannot extend up to infinity in such spacetimes [58,59], as we shall discuss below. In the inner Keplerian disc, the critical point of the vanishing angular-velocity gradient and its relation to the standard MRI viscosity mechanism is relevant, influencing thus the properties of the disc along the lines discussed in the previous case of the K-S naked singularity spacetimes. The creation 
of some toroidal structures can be thus relevant also for this reason.

\subsubsection{Class III: naked singularities with $\omega \in] \omega_{h}, \omega_{\gamma}[$}

The K-S naked singularity spacetimes containing two photon circular orbits, the inner stable one at $r_{\gamma}^{-}$, the outer unstable one at $r_{\gamma}^{+}>r_{\gamma}^{-}$, and one marginally stable orbit at $r_{m s o}^{+}>r_{\gamma}^{+}$. The stable circular orbits are located at $r \in] r_{\text {stat }}, r_{\gamma}^{-}\left[\right.$, and at $r>r_{m s o}^{+}$. Unstable circular orbits are located at $r \in] r_{\gamma}^{+}, r_{m s o}^{+}[$. At the region $r \in] r_{\gamma}^{-}, r_{\gamma}^{+}[$, no circular geodesics are allowed. There is no critical point related to the vanishing of the angular-velocity gradient. The angular velocity increases (decreases) with decreasing radius at the outer (inner) Keplerian disc.

In the outer Keplerian disc the viscosity MRI mechanism can work quite well and we can expect the standard Keplerian thermally radiating disc in the whole region above $r_{m s o}^{+}$. In the inner Keplerian disc some other viscosity mechanism could be working, however, its structure is quite nonstandard, as the energy and angular momentum diverge and change extremely fastly near the stable photon circular orbit, especially in the case of near-extreme $\mathrm{K}-\mathrm{S}$ naked singularity spacetimes. Creation of such a Keplerian structure by natural astrophysical processes seems to be very improbable - see [35].

\subsubsection{Class IV - black holes with $\omega>\omega_{h}$}

The K-S black hole spacetimes have the circular geodesics above the outer horizon only. The inner limit is given by the photon circular orbit $r_{\gamma}$. The unstable circular orbits are located between the photon circular orbit and the marginally stable orbit, $r_{m s o}^{+}$, above which the stable circular orbits are located, similarly to the case of the Schwarzschild spacetimes. Of course, the standard Keplerian thermally radiating disc with the MRI viscosity mechanism extends in the whole region above the ISCO.

\section{Structure of perfect fluid tori}

The structure and shape of the so-called test perfect fluid tori has been extensively studied for a variety of general relativistic spacetimes [49,51,58-64], and alternative gravity spacetimes, and in [68] a first superposition of a charged black hole with an annular disk made of extremal dust was presented, which could be compared to an alternative approach related to charged dust $[76,77]$. Here, the toroidal structures will be investigated in the $\mathrm{K}-\mathrm{S}$ naked singularity spacetimes of the modified Hořava quantum gravity, but the standard general relativistic methods of treating the test perfect fluid configu- rations will be applied, as they correspond to the IR end of the Hořava gravity.

The perfect fluid energy-momentum tensor components $T_{\mu \nu}$ relative to a coordinate basis read

$T_{\mu \nu}=(p+\rho) U_{\mu} U_{\nu}-p g_{\mu \nu}$

where $p(\rho)$ is the perfect fluid pressure (energy density) and $g_{\mu \nu}$ are the components of the metric tensor. The elements of the perfect fluid follow circular trajectories, i.e., their four-velocity, read $U^{\mu}=\left(U^{t}, 0,0, U^{\phi}\right)$. The Euler equation governing the structure and the shape of the perfect fluid configurations in a given spacetime can be cast in the form

$\frac{\nabla_{\mu} p}{p+\rho}=-\nabla_{\mu} \ln \left(U_{t}\right)+\frac{\Omega \nabla_{\mu} l}{1-\Omega l}$

where $\Omega(l)$ is the angular velocity (specific angular momentum) of the fluid element, defined by the relations

$\Omega=\frac{U^{\phi}}{U^{t}}, \quad l=-\frac{U_{\phi}}{U_{t}}$

For a barotropic fluid, as assumed here $[p=p(\rho)]$, it follows from Eq. (16) that there exists an invariant function $\Omega=\Omega(l)$ and surfaces of constant pressure are given by Boyer's condition [58]

$\int_{0}^{p} \frac{\mathrm{d} p}{p+\rho}=W(p)-W(0)=-\ln \frac{U_{t}}{\left(U_{t}\right)_{i n}}+\int_{l_{i n}}^{l} \frac{\Omega \mathrm{d} l}{1-\Omega l}$

To obtain a particular structure, we have to specify the functions $\Omega=\Omega(l)$ and $l=l(r, \theta)$. Usually, the simplest case of the marginally stable toroidal structures is studied, as it gives for the whole system substantial information on the structure of more complex toroidal configurations [58,61]. For a marginally stable torus, the specific angular momentum of the fluid element remains constant across the toroid, $l(r, \theta)=l_{0}=$ const. The angular velocity of the fluid then reads

$\Omega=-\frac{g_{t t}}{g_{\phi \phi}} l_{0}=\frac{f(r)}{r^{2} \sin ^{2} \theta} l_{0}$.

Under these assumptions, the function $W=W(r, \theta)$ takes the simple form

$W(r, \theta)=\frac{1}{2} \ln U_{t}^{2}=\frac{1}{2} \ln \left[\frac{f(r) r^{2} \sin ^{2} \theta}{r^{2} \sin ^{2} \theta-f(r) l_{0}^{2}}\right]$,

where $U_{t}$ follows from the normalization of the four-velocity $U^{\mu}$. The structure and shape of the perfect fluid equilibrium 
configurations are governed by the geodesic circular motion, as the pressure gradient vanishes at their center, which is governed by stable circular geodesics, while the cusps determining their edge where outflow of matter is possible from the equilibrium configurations are determined by unstable circular geodesics $[58,59]$. For this reason, the specific angular momentum of the geodesic motion, i.e. the angular momentum related to the energy, $l_{K} \equiv \frac{L_{K}}{E_{K}}$, plays an important role in constructing the equilibrium toroidal configurations. The shape of the equilibrium toroidal structures is given by the relation

$\frac{\mathrm{d} \theta}{\mathrm{d} r}=-\frac{\partial U_{t} / \partial r}{\partial U_{t} / \partial \theta}$

\section{Equilibrium tori in the K-S naked singularity spacetimes}

In the K-S spacetimes, the potential $W$ in Eq. (20) governing the equilibrium tori with uniform distribution of the specific angular momentum reads

$$
\begin{aligned}
& W(r, \theta ; l)=\frac{1}{2} \ln U_{t}^{2} \\
& =\frac{1}{2} \ln \frac{\left[1+r^{2} \omega\left(1-\sqrt{1+\frac{4}{\omega r^{3}}}\right)\right] r^{2} \sin ^{2} \theta}{r^{2} \sin ^{2} \theta-l^{2}\left[1+r^{2} \omega\left(1-\sqrt{1+\frac{4}{\omega r^{3}}}\right)\right]} .
\end{aligned}
$$

Its behavior in the equatorial plane is crucial, as it determines all the relevant properties of the equilibrium tori. In the equatorial plane, two conditions have to be satisfied for the reality of the potential $W$, namely

$$
\begin{aligned}
& f(r ; \omega)=1+r^{2} \omega\left(1-\sqrt{1+\frac{4}{\omega r^{3}}}\right) \geq 0, \\
& r^{2}-l^{2}\left(1+r^{2} \omega\left(1-\sqrt{1+\frac{4}{\omega r^{3}}}\right) \geq 0 .\right.
\end{aligned}
$$

The first condition means that the tori could be located in the stationary regions of the spacetime, while the second condition is clearly related to the photon geodesic motion, implying the condition

$$
l^{2}<l_{p h}^{2} \equiv \frac{r^{2}}{1+r^{2} \omega\left(1-\sqrt{1+\frac{4}{r^{3} \omega}}\right)} .
$$

The photon motion thus gives the reality condition of the equilibrium toroidal structures. The local extrema of the potential $W(r, \theta=\pi / 2)$ are given by the condition

$$
\begin{aligned}
l^{2} & =l_{K}^{2}(r, \omega) \equiv \frac{L_{K}^{2}(r ; \omega)}{E_{K}^{2}(r ; \omega)} \\
& =\frac{r^{2}\left[r^{3} \omega A(r, \omega)-\left(1+r^{3} \omega\right)\right]}{r A(r, \omega) f^{2}(r, \omega)},
\end{aligned}
$$

i.e., they are determined by the radial profile of the Keplerian specific angular momentum. Properties of the equilibrium tori are related to the properties of the circular geodesic motion. Therefore, classification of the $\mathrm{K}-\mathrm{S}$ spacetimes according to the properties of the equilibrium tori have to be analogous to the classification according to the circular geodesics summarized in Sect. 3.3. Of course, we have to distinguish also the $\mathrm{K}-\mathrm{S}$ spacetimes allowing for the existence of the marginally bound circular orbits with $E=1$. We give the relevant behavior of the functions $l_{K}^{2}(r, \omega)$ and $l_{p h}^{2}(r, \omega)$ in Fig. 2 for the values of the parameter $\omega$ characterizing all the classes of $\mathrm{K}-\mathrm{S}$ spacetimes.

\section{Classification of toroidal configurations in $\mathrm{K}-\mathrm{S}$ spacetimes}

In the case of $l=$ const tori, we can classify all the possible structures of the equipotential surfaces $W=$ const, and, thus, possible toroidal configurations of a perfect fluid, according to the values of $l$. Therefore, we present the behavior of the potential in the equatorial plane, i.e. the function $W(r, \theta=\pi / 2)$, and meridional sections through the structure of equipotential surfaces. We consider the square of the particle angular momentum $L^{2}$ and the fluid angular momentum $l^{2}$, as the potentials $V_{\text {eff }}(L)$ and $V_{\text {eff }}(l)$ are functions of $L^{2}$ or $l^{2}$, respectively, and the dynamics is independent of the orbital angular momentum sign. We concentrate our attention on the set $\mathscr{R} \equiv\left\{r_{m s o}^{ \pm}, r_{m b o}^{ \pm}, r_{\gamma}^{ \pm}, r_{\Omega}^{\text {Max }}, r_{\text {stat }}\right\}$, and the regions with boundaries in $\mathscr{R}$, the angular momenta $l_{i} \in \mathscr{L} \equiv\left\{l_{m s o}^{ \pm}, l_{m b o}^{ \pm}, l_{\gamma}^{ \pm}, l_{\Omega}^{\mathrm{Max}}, l_{\text {stat }}\right\}$ where $l_{i} \equiv l\left(r_{i}\right)$, and in general we adopt an analogous notation for any function $\mathbf{Q}(r): \mathbf{Q}_{i} \equiv \mathbf{Q}\left(r_{i}\right)$.

It is convenient to introduce the couple, with $C_{i}$ defined in Eq. (14),

$$
\begin{aligned}
\mathbf{C}_{c} & \equiv\left\{\omega_{c}, r_{c}\right\}, \quad \omega_{c} M^{2} \equiv \frac{1}{256}(37+30 \sqrt{3}) \\
& \approx 0.3475059, r_{c} \equiv \frac{8}{11}(2 \sqrt{3}-1) M \approx 1.79207 M
\end{aligned}
$$

where $r_{m b o}^{-}\left(\omega_{c}\right)=r_{\Omega}^{\operatorname{Max}}\left(\omega_{c}\right)=r_{c}$, and $r_{\text {stat }}<r_{\Omega}^{\operatorname{Max}}=$ $r_{m b o}^{-}<r_{m s o}^{-}<r_{m b o}^{+}<r_{m s o}^{+}$; see Fig. 1. We note, however, that the critical points of $\Omega(l)$ are related to the photon orbits for $\omega M^{2} \geq 2 / 3 \sqrt{3}$ as 

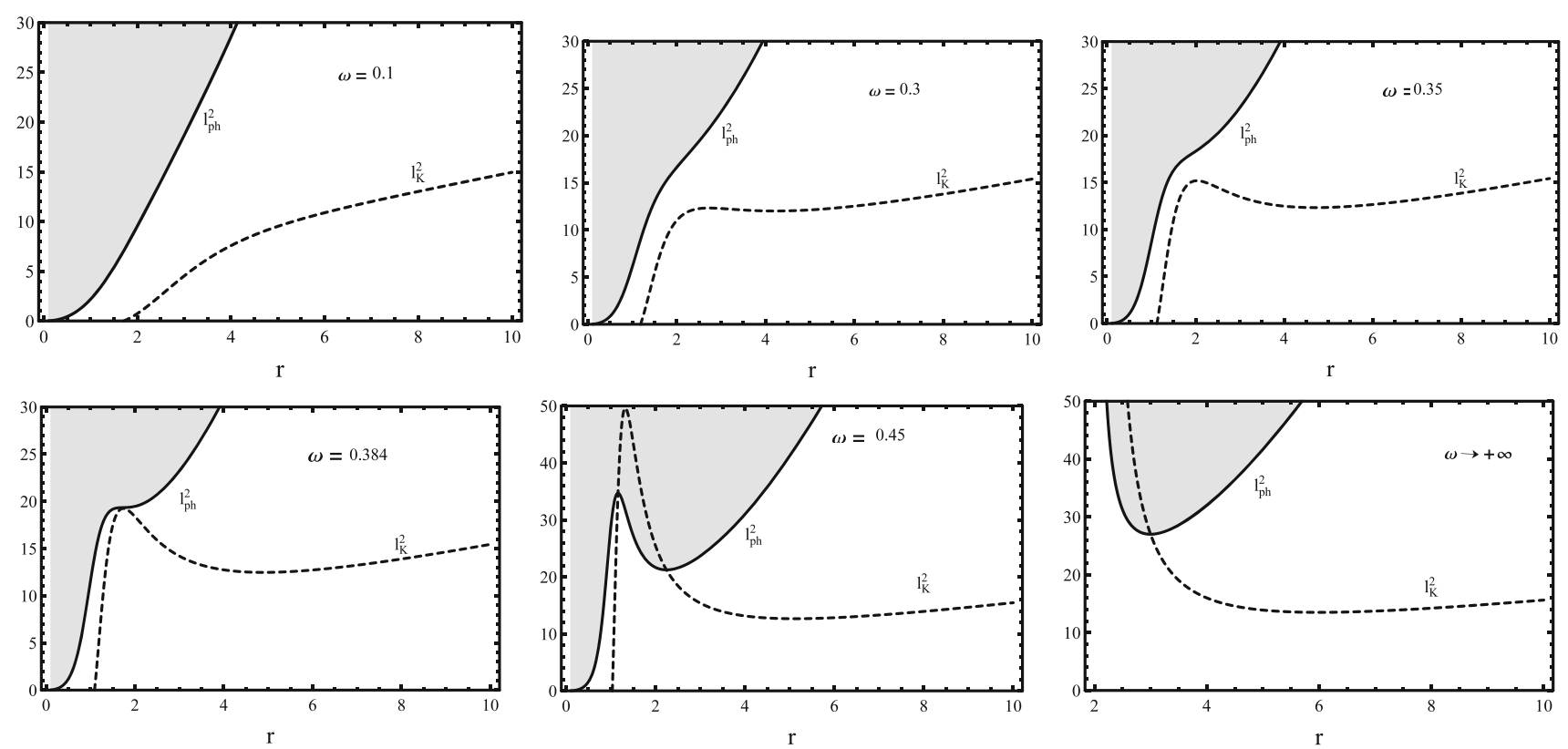

Fig. 2 Behavior of functions $l_{p h}^{2}$ and $l_{K}^{2}$, governing the equilibrium configurations with uniform distribution of the specific angular momentum, in five qualitatively different situations compared with the
Schwarzschild black hole case for values of the parameter $\omega$ characterizing all the classes of the $\mathrm{K}-\mathrm{S}$ spacetimes. The radius is in units of mass $M$ as $r / M \rightarrow r$ and also $\omega M^{2} \rightarrow \omega$

$$
\begin{aligned}
\Omega & =\frac{f(r)}{r^{2} \sin ^{2} \theta} l \\
l_{\Omega}^{\mathrm{Max}} & \equiv l\left(r_{\Omega}^{\mathrm{Max}}\right) \\
& =\sqrt{\frac{2^{2 / 3}(\sqrt{3}-2)}{4\left[(\sqrt{3}-1) \omega^{2 / 3}+2^{2 / 3}(\sqrt{3}-2) \omega\right]-2^{1 / 3} \omega^{1 / 3}}} .
\end{aligned}
$$

The angular momentum $l_{\Omega}^{\mathrm{Max}}$ has a critical point for $\omega M^{2}=\frac{1}{432}(5+3 \sqrt{3})=0.0236022-$ Fig. 1 . We shall consider separately the naked singularity case $\left(\omega M^{2} \in\right.$ ]0, 1/2[) in Sect. 6.1 and the black hole case $\left(\omega M^{2}>1 / 2\right)$ in Sect. 6.3. The extreme BH case $\left(\omega M^{2}=1 / 2\right)$ is addressed in Sect. 6.2.

\subsection{Naked singularity case: $\left.\omega M^{2} \in\right] 0,0.5[$}

In the $\mathrm{K}-\mathrm{S}$ naked singularity spacetimes it is convenient to analyze the four regions of $\omega$ and correspondingly four classes of naked singularity sources, namely as follows. Region I: $\omega \in]$ 0, $\omega_{m s o}$ [, explored in Sect. 6.1.1, corresponds to the naked singularities in Class III of Sect. 3.3.1, Region II: $\omega \in] \omega_{m s o}, \omega_{m b o}[$ in Sect. 6.1.3, Region IIIa: $\omega \in] \omega_{m b o}, \omega_{c}[$ in Sect. 6.1.5, Region IIIb: $\omega \in] \omega_{c}, \omega_{\gamma}[$ in Sect. 6.1.7. The set of geometries of Region II-IIIb corresponds to the spacetimes of Class II discussed in Sect. 3.3.2. Finally Region IV: $\omega \in] \omega_{\gamma}, 0.5$ [, considered in Sect. 6.1.9, corresponds to naked singularities of Class III discussed for the Keplerian orbits profile in Sect. 3.3.3. The particular cases are considered in the following way: $\omega=\omega_{m s o}$ in Sect. 6.1.2, $\omega=\omega_{m b o}$ in Sect. 6.1.4, $\omega=\omega_{c}$ in Sect. 6.1.6, and $\omega=\omega_{\gamma}$ in Sect. 6.1.8.

\subsubsection{Region I: $\omega \in] 0, \omega_{m s o}[$}

We focus on the naked singularity sources at $\omega \in] 0, \omega_{m s o}[$ where $\omega_{m s o} M^{2}=0.2810998$ and $r_{m s o}^{ \pm}\left(\omega_{m s o}\right)=3.51399 M$. The properties of the circular motion of the test particle have been detailed in Sect. 3.3.1. There is then no last stable circular orbit or photon circular orbit and no unstable circular orbit. In this region we have $l_{\Omega}^{\mathrm{Max}}>l_{\text {stat }}$ and $r_{\text {stat }}<r_{\Omega}^{\mathrm{Max}}$. Only stable circular orbits for $V_{\text {eff }}(L)$ are allowed. Since all the circular geodesic orbits are stable, it is possible to consider the Keplerian discs centered in $r \in] r_{\text {stat }},+\infty[$. In Region I no unstable modes are possible. We summarize the situation as follows:

$-l>l_{\Omega}^{\mathrm{Max}}$ The radius $r_{\Omega}^{\mathrm{Max}}$ determines the location of the critical points of the angular frequency $\Omega(r, L)$. For fluid angular momentum $l>l_{\Omega}^{\mathrm{Max}}$ there are always stable orbits with $V_{\text {eff }}(l, r)<1$. This minimum of the effective potential determines the location of the disc center; see Fig. 3a.

$-l=l_{\Omega}^{\mathrm{Max}}$ In this particular case the center of the disc (maximum of the hydrostatic pressure) is located on $r_{\Omega}^{\mathrm{Max}}$ and the inner edge of the disc is in the region $r<r_{\Omega}^{M a x}$, the discs morphology is analogous to the situation in Fig. $3 \mathrm{a}$ 

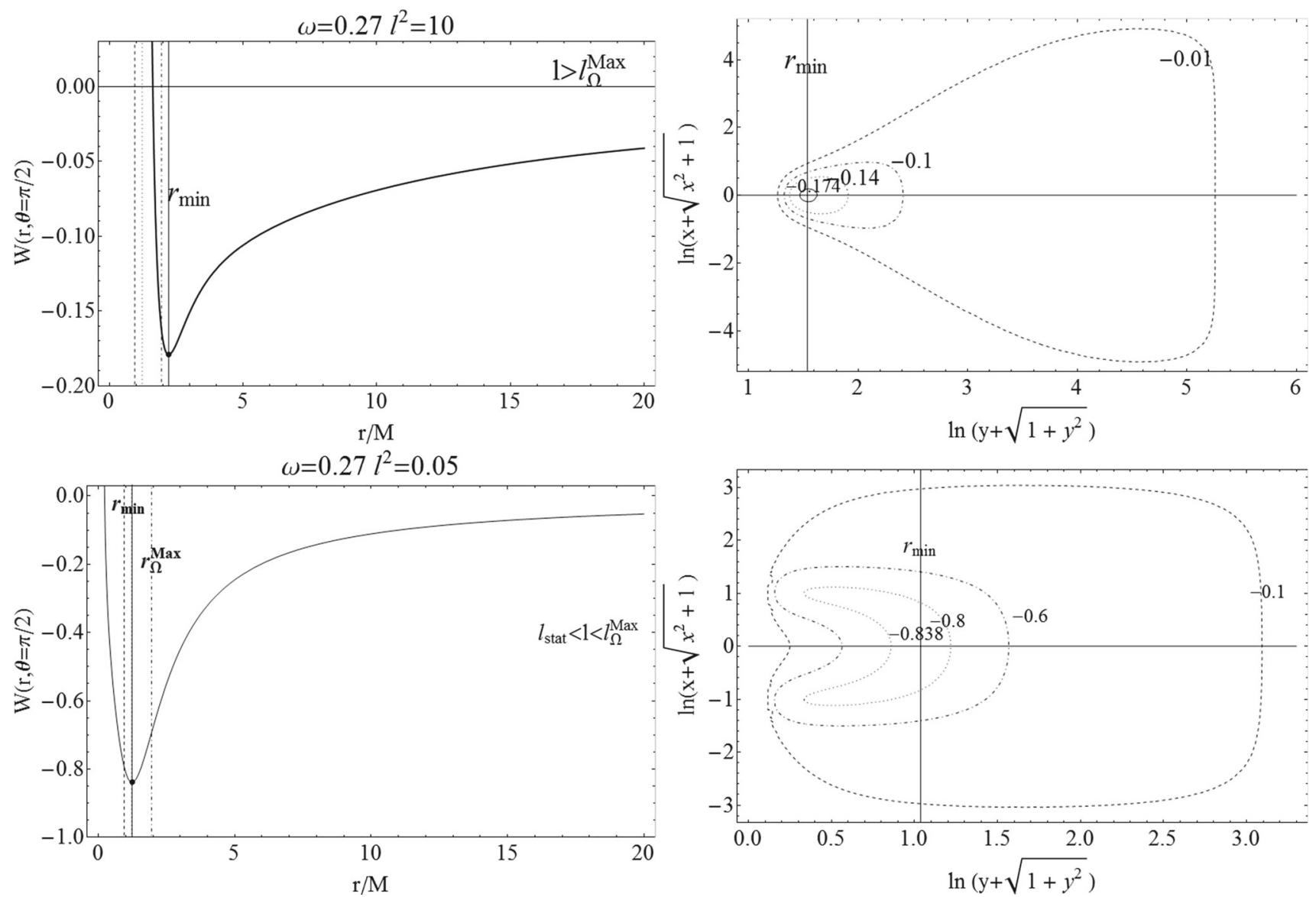

Fig. 3 Region I: $\omega \in] 0, \omega_{m s o}\left[\right.$, naked singularity $\omega M^{2}=0.27$. We have $l_{\Omega}^{\mathrm{Max}}>l_{\text {stat }}$, and $r_{\text {stat }}<r_{\Omega}^{\mathrm{Max}}$. The radius is in units of mass $M$ as $r / M \rightarrow r$ and $\omega M^{2} \rightarrow \omega$. We have $r / M=$

for $l>l_{\Omega}^{\mathrm{Max}}$. An analogous situation occurs for the following case.

$-l \in] l_{\text {stat }}, l_{\Omega}^{\mathrm{Max}}$ ] See Fig. 3b. As $l$ decreases approaching the limit $l \approx 0$, the closed surfaces thicken approaching the sources.

\subsubsection{Naked singularity: $\omega=\omega_{m s o}$}

In this section we analyse the naked singularity geometry with $\omega=\omega_{m s o}$, separating geometries with two marginally stable radii $r_{m s o}^{ \pm}$(Region $I I-I V$ ) and the class of a spacetime with a naked singularity where no marginally stable orbits are allowed (Region I). Then $r_{\text {stat }}<r_{\Omega}^{\mathrm{Max}}<r_{m s o}^{-}=r_{m s o}^{+}$ and $l_{m s o}^{-}=l_{m s o}^{+}>l_{\Omega}^{\mathrm{Max}}>l_{\text {stat }}$.

$-l>l_{m s o}$ Only minimum points of the effective potential are possible and closed fluid surfaces centered on the minimum points Fig. 3.

$-l=l_{m s o}$ The critical point of the effective potential is located on $r_{m s o}$, and for $\left(r=r_{m s o}, l=l_{m s o}\right)$ we have $V=0.925491, \partial_{r} V=0$, and $\partial_{r}^{(2)} V=0$. The configu-

$\sqrt{x^{2}+y^{2}}$ where $(x, y)$ are Cartesian coordinates. Vertical lines in the right panels set the $r_{i} \in \mathscr{R}$ and the effective potential critical points

ration for $l \approx l_{m s o}$ is analogous to the case of $l>l_{m s o}$ in Fig. 3.

- $l \in] l_{\text {stat }}, l_{m s o}[$ This case includes the ranges $l \in] l_{\text {stat }}, l_{\Omega}^{\mathrm{Max}}$ $\left[\cup\left[l_{\Omega}^{\mathrm{Max}}, l_{m s o}[\right.\right.$. There are only closed surfaces centered in $r_{\min }$ as in Fig. 3.

\subsubsection{Region II: $\omega \in] \omega_{m s o}, \omega_{m b o}[$}

The K-S naked singularity spacetimes of Region II contain no photon circular orbits or marginally bounded orbits, but two marginally stable circular orbits at $r_{m s o}^{ \pm}: r_{m s o}^{-}<r_{m s o}^{+}$, respectively. We have $r_{\text {stat }}<r_{\Omega}^{\mathrm{Max}}<r_{m s o}^{-}<r_{m s o}^{+}$and $l_{m s o}^{-}>l_{m s o}^{+}>l_{\Omega}^{\mathrm{Max}}>l_{\text {stat }}$. As detailed in Sect. 3.3.2 there are stable circular orbits in $r_{\text {min }}^{+}>r_{m s o}^{+}$(which corresponds to the outer $C^{+}$disc) and $\left.r_{\min }^{-} \in\right] r_{\text {stat }}, r_{m s o}^{-}$[ (which corresponds to the center of the inner $C^{-}$disc). Unstable circular orbits are in $\left.r_{\text {Max }} \in\right] r_{m s o}^{-}, r_{m s o}^{+}$[. In $r_{\text {stat }}$ we have $l_{\text {stat }}=0$, $\left.V_{\text {stat }} \in\right] 0,1\left[, \partial_{r} V_{\text {stat }}=0\right.$, and $\partial_{r}^{2} V_{\text {stat }}>0$.

As two disconnected regions of orbital stability characterize these spacetimes, the existence of a double family of closed configurations is foreseen. An outer $C^{+}$with cen- 
ter in $r_{\min }^{+}$and an inner one $C^{-}$centered in $r_{\min }^{-}$. Since $r_{\text {min }}^{-}<r_{\text {Max }}<r_{\text {min }}^{+}$, an instability point, cross for the disc surface, occurs in between the two families of closed surfaces. As $V_{\text {Max }}<1$, the crossed surface is closed as well, having two lobes. No accretion into the singularity is possible. This is a characteristic feature of all the K-S naked singularity solutions. Matter is prevented to accrete the singularity, thus one can say that the naked singularity is embedded in a region of "fluid-impermeable" vacuum.

For $\omega>\omega_{m b o}$, excretion of matter can occur. This phenomenon is interpreted as a repulsive gravity effect, and it characterizes in general all geometries with naked singularities. The cusp enables outflow of fluid from one disc to the other by violation of hydrostatic equilibrium, and giving rise to a feeding between the discs: the outer edge of the inner torus is located above the (outer) marginally stable circular geodesic, i.e. $r_{m s o}^{-}$. Just as in a binary system one disc is fed by the outcome of matter from the companion. The absence of an accretion point for this class of $\mathrm{K}-\mathrm{S}$ naked singularity could be compared with the analog cases where the gravitational attraction is balanced for example by the cosmic repulsion due to a cosmological constant, for instance in relativistic thick discs in the Kerr-de Sitter backgrounds [62] or in the Reissner-Nordström-anti-de Sitter [51]. It should be noted that these last cases can be associated to effective potential functions admitting two cups due to double unstable points separated by a stability region where the center of the disc is located. The crossed configuration allows accretion through the inner cusp and/or excretion through the outer cusp.

In the present case a double configuration is possible only in a specific set of $\mathrm{K}-\mathrm{S}$ naked singularities, namely those with critical parameter $\omega$ sufficiently close to the extreme black hole case, i.e. the sources belonging to Region $I I-I V$, the repulsive effect is due to a combination of the "antigravity" effect typical of the NS-cases and the centrifugal repulsion. The main difference with the aforementioned cosmological spacetimes is that for a $\mathrm{K}-\mathrm{S}$ naked singularity there are no double cusp configurations, but only double centered ones. Two main aspects of the effective potential function regulate the excretion of matter and the feeding from one disc to the other as well as other essential characteristics of the torus morphology such as the thickness, namely: the presence of (also stable) orbits at $E>1$ (characterizing spacetimes in Region $I I I-I V$ ) and the gap between the $W$-values as in the two minimum points. Three cases should be distinguished: $I$ for $W_{\min }^{-}>W_{\min }^{+}, I I$ for $W_{\min }^{-}=W_{\min }^{+}$, and $I I I$ for $W_{\min }^{-}<W_{\min }^{+}$, and correspondingly three types of configurations belonging to the cases $I-I I-I I I$ should be considered, respectively. As the outer minimum is always negative (see the location of the orbits $r_{m b o}^{ \pm}$, compared to $r_{m s o}^{ \pm}$in Fig. 1, the case in which the interior minimum is positive or zero is easily located in the class $I$ (for $W>0$ in $r_{m s o}<r_{\min }^{i}<r_{m b o}$ ). $-l>l_{m s o}^{-}$There are closed stable configurations. The effective potential has only one minimum point and no maxima; see Fig. 3.

$-l=l_{m s o}^{-}$This case is illustrated in Fig. 4: at $r_{m s o}^{-}$there is a saddle point of the effective potential, a minimum is located at $r_{\min }>r_{m s o}^{+}$. There is only a closed configuration. The value $W_{m s o}^{+}$is actually a critical point, corresponding to the inner edge of the disc centered in $r_{\text {min. }}$. As in Fig. 4 we have $\left|W_{m s o}^{-}\right|<\left|W_{m s o}^{+}\right|$, the maximum value of $W$ for the appearance of the thick torus is $\left|W_{\text {min }}\right|$. As $W \gtrsim W_{\text {min }}^{+}$a sequence of centered configurations starts, which thickens with increasing $W$ until the values $W_{m s o}^{-}$where a cusped inner edge is formed, and then another closed configuration filled the region.

$-l \in] l_{m s o}^{+}, l_{m s o}^{-}[$There are two minimum and one maximum points: $r_{\text {stat }}<r_{\text {min }}^{-}<r_{\text {mso }}^{-}<r_{\text {max }}<r_{\text {mso }}^{+}<r_{\text {min }}^{+}$. As a consequence of this, there is a double closed configuration of one inner torus $C^{-}$centered in $r_{\min }^{-}$and $C^{+}$ in $r_{\min }^{+}$(the outer one). As $W$ increases toward $W_{\text {Max }}$, the outer edge of $C^{-}$approaches the inner edge of $C^{+}$until a double centered, closed crossed configuration appears for $W_{\text {Max }}$. As $W$ approaches asymptotically $W=0$, for $W \in] W_{\text {Max }}, 0[$ only one closed configuration appears with two thickness maxima in $r_{\text {min }}$. In Fig. 4 , the case $I$ is shown. The maximum value of $|W|$ for the formation of a thick configuration is $\left|W_{\min }^{+}\right|$: the outer configuration $C^{+}$is formed, and as $W$ fills the gap $\Delta=\left|W_{\min }^{+}-W_{\min }^{-}\right|$, a second $\operatorname{disc} C^{-}$appears, thickening with increasing $W$ until $W=W_{\max }$.

$-l=l_{m s o}^{+}$This case is discussed in Fig. 5. At $r_{m s o}^{+}$a saddle point of the potential $W$ is located. A minimum is located in $r_{\min }^{-}<r_{m s o}^{-}$, which corresponds to the center of the closed configuration. A closed (stable) disc is formed as $W \gtrsim W_{\min }^{-}$. For $W=W_{m s o}^{+}$a configuration similar to that in Fig. 4 occurs with $l=l_{m s o}^{-}$, where the center of torus is in $r_{\min }^{+}$. We stress that the location of the "cusp" in the two cases is reversed. With increasing $W>W_{m s o}^{+}$one closed stable configuration with two maximum points of thickness appears. Finally we could treat the peculiar case shown in Fig. 4 where only one minimum and a saddle point occur in III for $W_{\min }^{-}<W_{m s o}^{+}$.

$-l \in] l_{\text {stat }}, l_{m s o}^{+}[$This range includes $] l_{\text {stat }}, l_{m s o}^{+}[=] l_{\text {stat }}, l_{\Omega}^{\mathrm{Max}}$ $\left[\cup\left[l_{\Omega}^{\mathrm{Max}}, l_{m s o}^{+}[\right.\right.$. There are only closed, stable configurations with center located in $r=r_{\min }^{+}$. This case is similar to the analogous situation for attractors in Region I; see Fig. 3.

\subsubsection{Naked singularity: $\omega=\omega_{m b o}$}

In this section we consider the particular case of a naked singularity spacetime with $\omega=\omega_{m b o}$ where $r_{\text {stat }}<r_{\Omega}^{\operatorname{Max}}<$ 

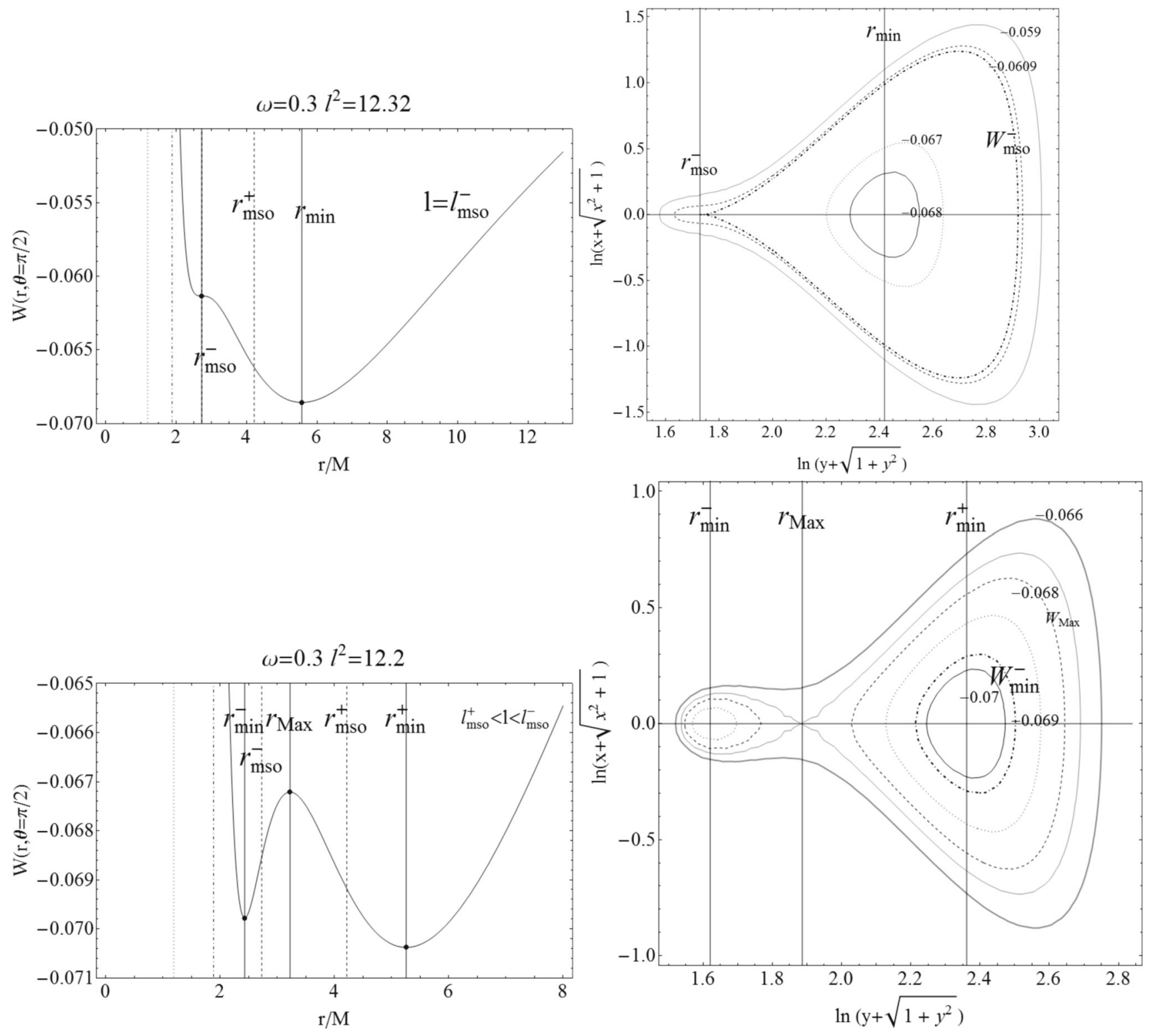

Fig. 4 Region II: $\omega \in] \omega_{m s o}, \omega_{m b o}\left[\right.$, naked singularity $\omega M^{2}=0.3$. Here $\omega M^{2} \rightarrow \omega$. Vertical lines in the right panels set the $r_{i} \in \mathscr{R}$ and the effective potential critical points. We have $l_{m s o}^{-}>l_{m s o}^{+}>l_{\Omega}^{\mathrm{Max}}>l_{\text {stat }}$,

and $r_{\text {stat }}<r_{\Omega}^{\operatorname{Max}}<r_{m s o}^{-}<r_{m s o}^{+}$, where $r / M=\sqrt{x^{2}+y^{2}}$ and $(x, y)$ are Cartesian coordinates

$-l=l_{m s o}^{-}$This case is illustrated in Fig. 6. The effective potential has a minimum $W_{\min }<0$ in $r_{\min }>r_{m s o}^{+}$. At $r=r_{m s o}^{-}=r_{m b o}$, the effective potential has a saddle point where $W_{m s o}^{-}=0$, correspondingly a set of closed configurations centered in $r_{\min }$ occurs for $\left.W \in\right] W_{\min }, 0[$, being more and more stretched, at the equatorial plane as $W$ approaches the limit $W=W_{m s o}^{-}$where an open surface appears.

$-l \in] l_{\Omega}^{\mathrm{Max}}, l_{m s o}^{-}[$There are three critical points of the hydrostatic pressure for $W_{\text {crit }}<0$. Correspondingly, there are generally two closed configurations $C^{ \pm}$centered in $r_{\min }^{-}<r_{\min }^{+}$, respectively, the closed-cross sur- 


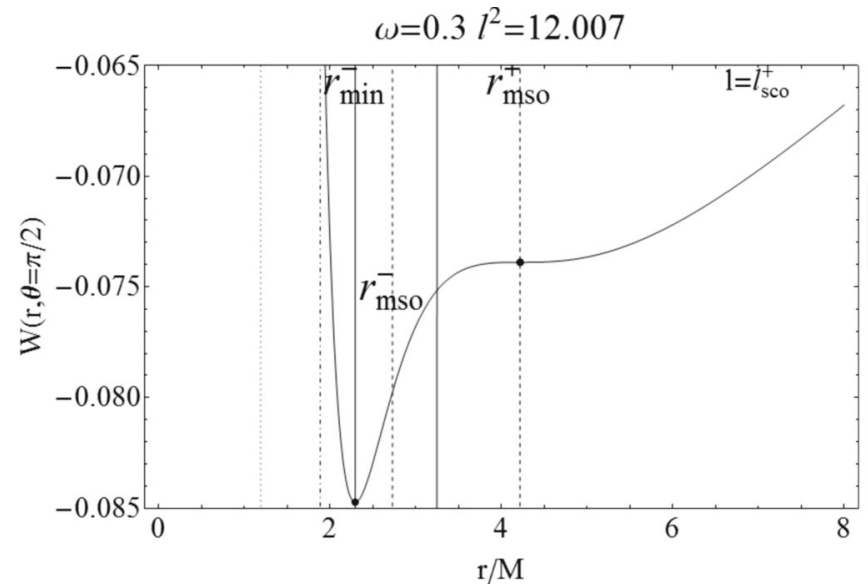

Fig. 5 Region II: $\omega \in] \omega_{m s o}, \omega_{m b o}\left[\right.$, naked singularity $\omega M^{2}=$ 0.3 . Here $\omega M^{2} \rightarrow \omega$. We have $l_{m s o}^{-}>l_{m s o}^{+}>l_{\Omega}^{\mathrm{Max}}>$ $l_{\text {stat }}$, and $r_{\text {stat }}<r_{\Omega}^{\mathrm{Max}}<r_{m s o}^{-}<r_{m s o}^{+}$, where $r / M=$
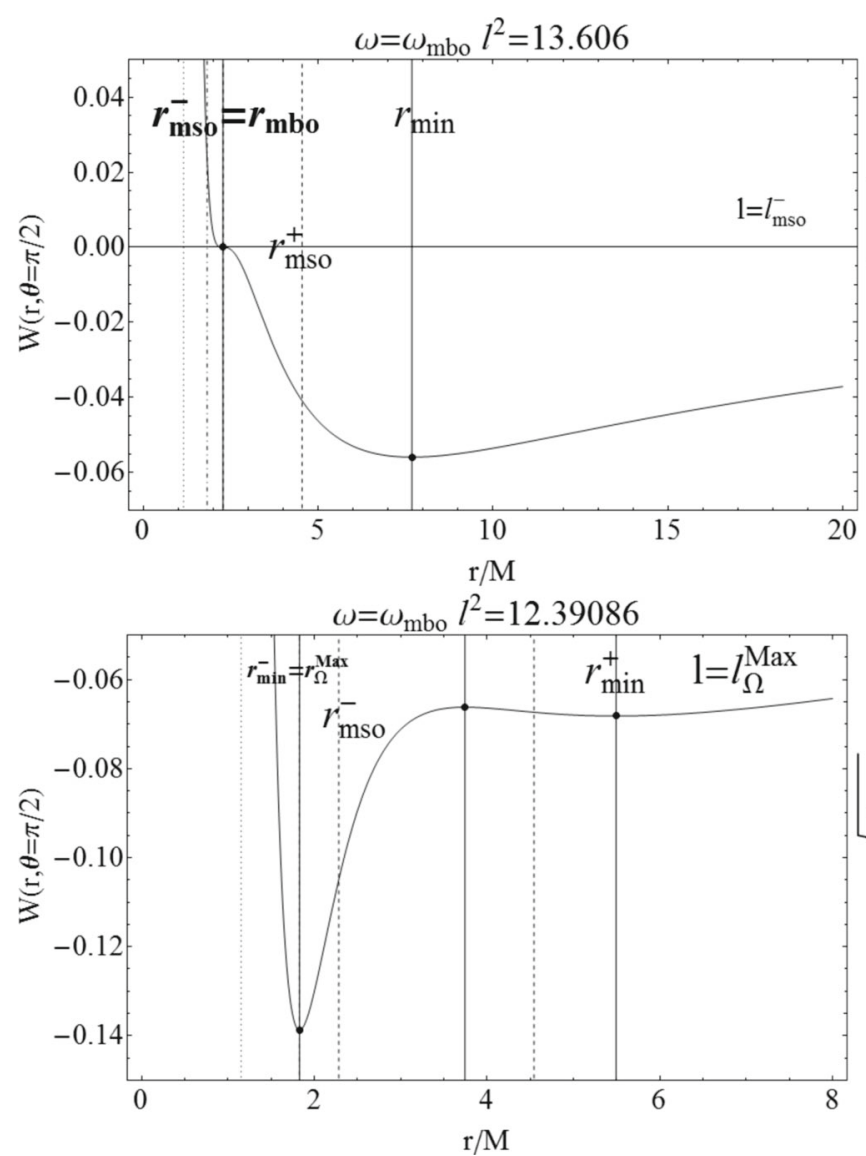

Fig. 6 Naked singularity $\omega=\omega_{m b o}$. We have $l_{m s o}^{-}=l_{m b o}^{-}=l_{m b o}^{+}>$ $l_{\Omega}^{\mathrm{Max}}>l_{m s o}^{+}$, and $r_{\text {stat }}<r_{\Omega}^{\mathrm{Max}}<r_{m b o}^{-}=r_{m s o}^{-}=r_{m b o}^{+}<r_{m s o}^{+}$. Vertical lines in the right panels set the $r_{i} \in \mathscr{R}$ and the effective potential critical

face occurs for $W=W_{\text {Max }}<0$. This case is analogous to the case in Fig. 3 for singularities in Region II with $l \in] l_{m s o}^{+}, l_{m s o}^{-}[$. This is a double III configuration, as $W_{\min }^{-}<W_{\min }^{+}$.

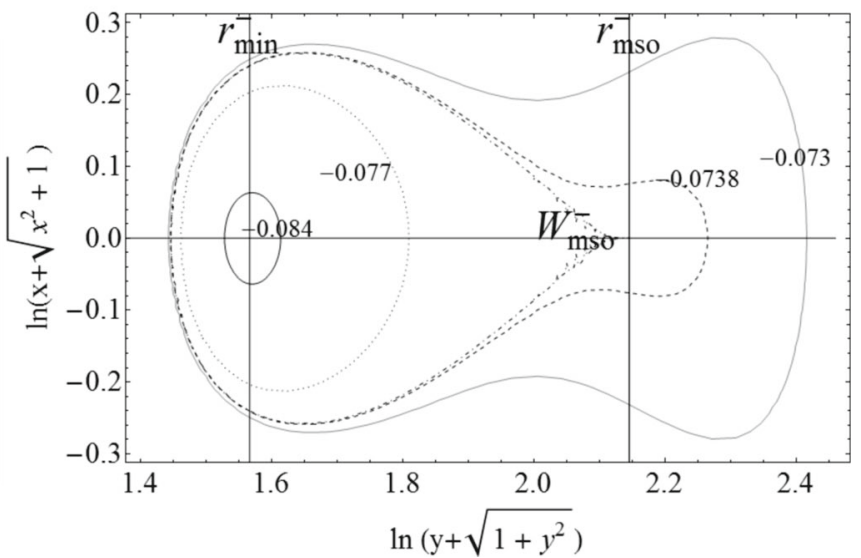

$\sqrt{x^{2}+y^{2}}$ and $(x, y)$ are Cartesian coordinates. Vertical lines in the right panels set the $r_{i} \in \mathscr{R}$ and the effective potential critical points
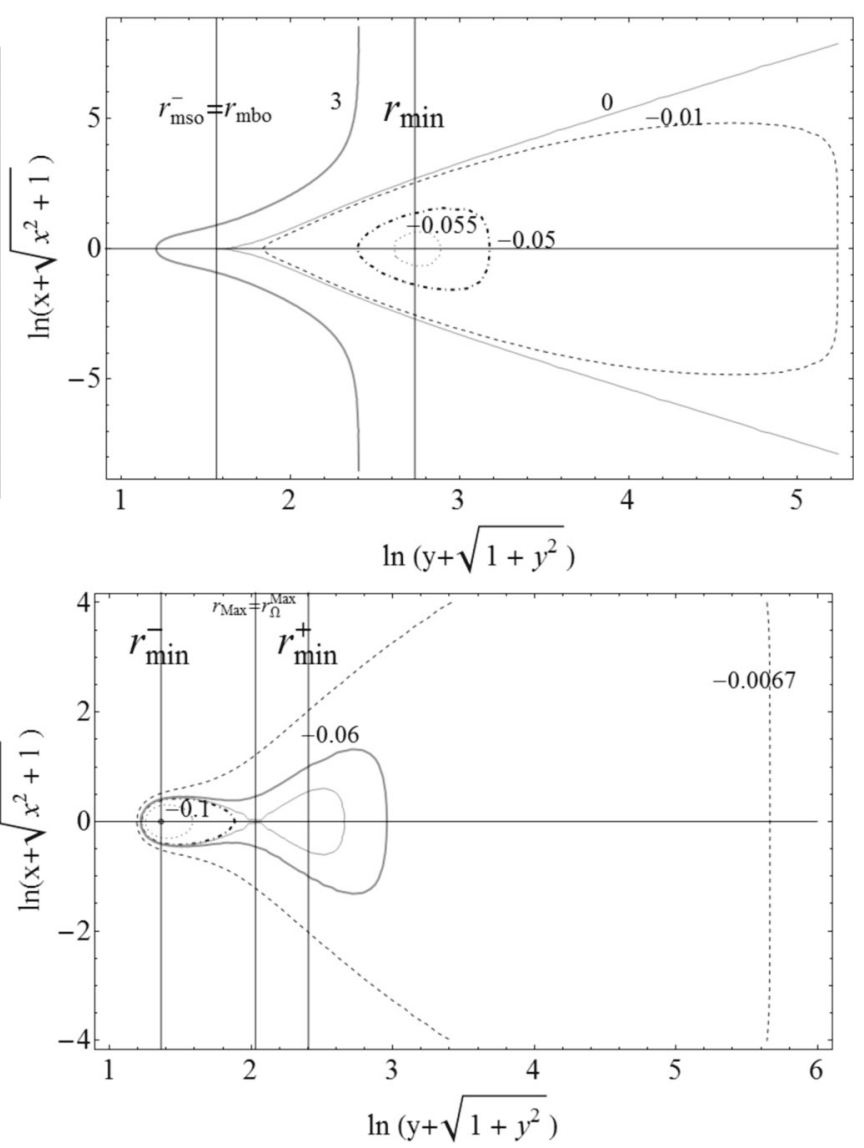

points. We have $\omega M^{2} \rightarrow \omega$, where $r / M=\sqrt{x^{2}+y^{2}}$ and $(x, y)$ are Cartesian coordinates

$-l=l_{\Omega}^{\mathrm{Max}}$ There is a minimum of the effective potential in $r_{\Omega}^{\text {Max }}<r_{m s o}^{-}$, a minimum $r_{\text {min }}^{+}>r_{m s o}^{+}$and a maximum point $r_{\text {Max }}$. The situation is similar to the case $l \in] l_{\Omega}^{\mathrm{Max}}, l_{m b o}[$; see Fig. 6 . The situation is analogous to 
the case $l \in] l_{\Omega}^{\mathrm{Max}}, l_{m s o}^{-}$[ and leads to a double III configuration. The torus thickness, the spatial distances between the inner edge of the outer torus and the outer edge of the inner configuration, are regulated by the magnitude of the effective potential $W$ and the energy gap $\left|W_{\min }^{+}-W_{\min }^{-}\right|$.

$-l \in] l_{m s o}^{+}, l_{\Omega}^{\mathrm{Max}}\left[\right.$ There are closed $C^{ \pm}$, type-III toroidal surfaces and a critical surface. The behavior of the equipotential surfaces is analogous to the situation in Fig. 6.

$-l=l_{m s o}^{+}$There is a sequence of type $I I I$ of closed $C^{-}$ toroidal surfaces centered in $r_{\text {min }}^{-}$and a critical closed (but not crossed) surface corresponding to the saddle point of the effective potential. As this critical point is located in $r_{m s o}^{+}$, the "cusp" is directed toward the outer space, and the situation is similar to other cases where $l=l_{m s o}^{+}$, for example in Fig. 5.

$-l \in] l_{\text {stat }}, l_{m s o}^{+}\left[\right.$There are only closed $C^{-}$(stable) type-III configurations centered in $r_{\min }<r_{m s o}^{-}$; see Fig. 3.

\subsubsection{Region IIIa: $\omega \in] \omega_{m b o}, \omega_{c}[$}

In Kehagias-Sfetsos naked singularity spacetimes with Hořava parameter in Region IIIa the situation is as follows: $r_{\text {stat }}<r_{\Omega}^{\mathrm{Max}}<r_{m b o}^{-}<r_{m s o}^{-}<r_{m b o}^{+}<r_{m s o}^{+}$where $l_{m s o}^{-}>l_{m b o}^{-}>l_{m b o}^{+}>l_{\Omega}^{\mathrm{Max}}>l_{m s o}^{+}$and $W_{m b o}^{ \pm}=0$. Naked singularities in Region III- $a$ belong to the attractors of Class II, and therefore the structure of the test particle circular motion has been discussed in Sect. 3.3.2. Toroidal configurations in these naked singularity spacetimes are characterized by the presence of the excretion points. In detail:

$-l>l_{m s o}^{-}$There are only stable toroidal surfaces as in Fig. 3.

$-l=l_{m s o}^{-}$There is a saddle point located in $r_{m s o}^{-}$, see Fig. 7a, where the effective potential $W_{m s o}>0$. A minimum is at $r_{\min }^{+}>r_{m s o}^{+}$: toroidal stable configurations are as $W \in] W_{\min }^{+}, 0[$. Only one "critical" open surface is located in $r_{m s o}^{-}$, which corresponds to an "inner cusp". We can compare this situation to the case $l=l_{m s o}^{-}$at $\omega=\omega_{m s o}$ (see also Fig. $3 \mathrm{~b}$ ) where $W_{m s o}^{-}=0$. No accretion into the singularity is allowed in any case.

$-l \in] l_{m b o}^{-}, l_{m s o}^{-}$[ The inner minimum is located at $r_{\min }^{-}$: $W_{\min }^{-}>0$. There is a maximum $r_{\text {Max }} W_{\mathrm{Max}}>0$, and the outer minimum is at $r_{\min }^{+}: W_{\min }^{+}<0$. A toroidal set of closed and stable configurations is at $W \in] W_{\min }^{+}, 0[$ : as the potential increases the surface stretches along the equatorial plane and thickens approaching the asymptotic superior limit of $W=0$. A further set of inner closed $C^{-}$configurations is centered at $r_{\min }^{-}$: since $\left(W_{\min }^{-}>\right.$ $\left.0, W_{\min }^{+}<0\right)$, there are no double closed configurations at equal $W$ and $l$. According to the tori classification proposed for configurations with $l \in] l_{m s o}^{+}, l_{m s o}^{-}$[ in Region II (Fig. 3), the torus model illustrated in Fig. 7b is consid- ered to be of type $I$, because of $W_{\min }^{-}>W_{\min }^{+}$. As long as $W$ increases toward its maximum, an outer cusp appears, leading to a crossed point, opened toward the exterior, allowing for the excretion of matter. Finally we note that the outer (stable) configuration can be larger and thicker than the inner (unstable and excreting) one. The thickness and other geometrical torii features are regulated by the gaps $\Delta_{i} \equiv\left|W_{\min }^{-}-W_{\text {Max }}\right|<\Delta_{o} \equiv\left|W_{\min }^{+}-0\right|$ and $\Delta_{i}^{r} \equiv\left|r_{\min }^{-}-r_{\text {Max }}\right|$, and the location of the crossing points $r_{x}^{o}:\left.W\left(r_{x}^{o}\right)\right|_{\theta=\pi / 2}=0$ and $r_{x}^{i}:\left.W\left(r_{x}^{i}\right)\right|_{\theta=\pi / 2}=W_{\min }^{-}$.

$-l=l_{m b o}^{-}$This case is illustrated in Fig. 7c, is a limiting case with respect to Fig. $7 \mathrm{~b}$, where $W_{\min }^{-}=0$. It could also be compared to the configurations with $l=l_{m b o}^{-}$, for $\omega=\omega_{m s o}$ as illustrated in Fig. 3.

$-l \in] l_{m b o}^{+}, l_{m b o}^{-}$[illustrated in Fig. 8a. The values $W_{\min }^{-}<0$ and $W_{\text {Max }}>0$ decrease with the fluid's angular momentum increasing. There is one closed stable configuration for $W \in] W_{\min }^{+}, W_{\min }^{-}[$, but there are double closed stable configurations for $W \in] W_{\min }^{-}, 0[$, the value $W=0$ corresponds to a closed configuration $C^{-}$centered in $r_{\min }^{-}$, and an open outer one, for $W \in] 0, W_{\operatorname{Max}}[$. There are closed stable $C^{+}$toroidal configurations with center in $r_{\text {min }}^{+}$. Finally, at $W_{\text {Max }}$ a crossed surface with an outer cusp occurs, being associated with the excretion. These are type-I configurations.

$-l=l_{m b o}^{+}$In this case the maximum of the configuration is located at $r_{\text {mbo }}^{+}$as such a torus has $W_{\text {max }}=0$. The situation, sketched in Fig. 8b, is qualitatively similar to Fig. 8a. This is a type-I configuration.

$-l \in] l_{\Omega}^{\mathrm{Max}}, l_{\text {mbo }}^{+}$Now $W_{\text {Max }}<0$. At $W=W_{\text {Max }}$, there is a double centered crossed closed configuration, Fig. 8c, and this is a type-I configuration.

$-l=l_{\Omega}^{\mathrm{Max}}$ Now $r_{\min }^{-}=r_{\Omega}^{\mathrm{Max}}$, consequently, there is an inner set of closed $C^{-}$surfaces centered in $r_{\Omega}^{\mathrm{Max}}$; see Fig. 6. In this case there is $W_{\min }^{-}<W_{\min }^{+}<0$, therefore this is a $I I I$-configuration. There is a continuous shift between type- $I$ and type-III configurations with the $I I$ type having $W_{\min }^{-}=W_{\min }^{+}<0$. There are no excretion points.

$-l \in] l_{m s o}^{+}, l_{\Omega}^{\mathrm{Max}}[$ This torus is analogous to Fig. 6; there is a couple $C^{ \pm}$of closed III configurations.

$-l=l_{m s o}^{+}$There is a saddle point located in $r_{m s o}^{+}$, implying a critical configuration, the "outer cusp", as in Fig. 5.

$-l \in] l_{\text {stat }}, l_{m s o}^{+}\left[\right.$There is only one minimum in $r_{\text {min }}^{-}$, and correspondingly one set of closed $C^{-}$toroidal configurations centered in $r_{\text {min }}^{-}$; see Fig. 3.

\subsubsection{Naked singularity: $\omega=\omega_{c}$}

We focus on the special $\mathrm{K}-\mathrm{S}$ naked singularity case spacetime $\omega_{c} M^{2}=0.347506$, where $r_{\text {stat }}<r_{m b o}^{-}=r_{\Omega}^{\mathrm{Max}}<r_{m s o}^{-}<$ $r_{m b o}^{+}<r_{m s o}^{+}$and $l_{m s o}^{-}>l_{\Omega}^{\mathrm{Max}}=l_{m b o}^{-}>l_{m b o}^{+}>l_{m s o}^{+}$. 

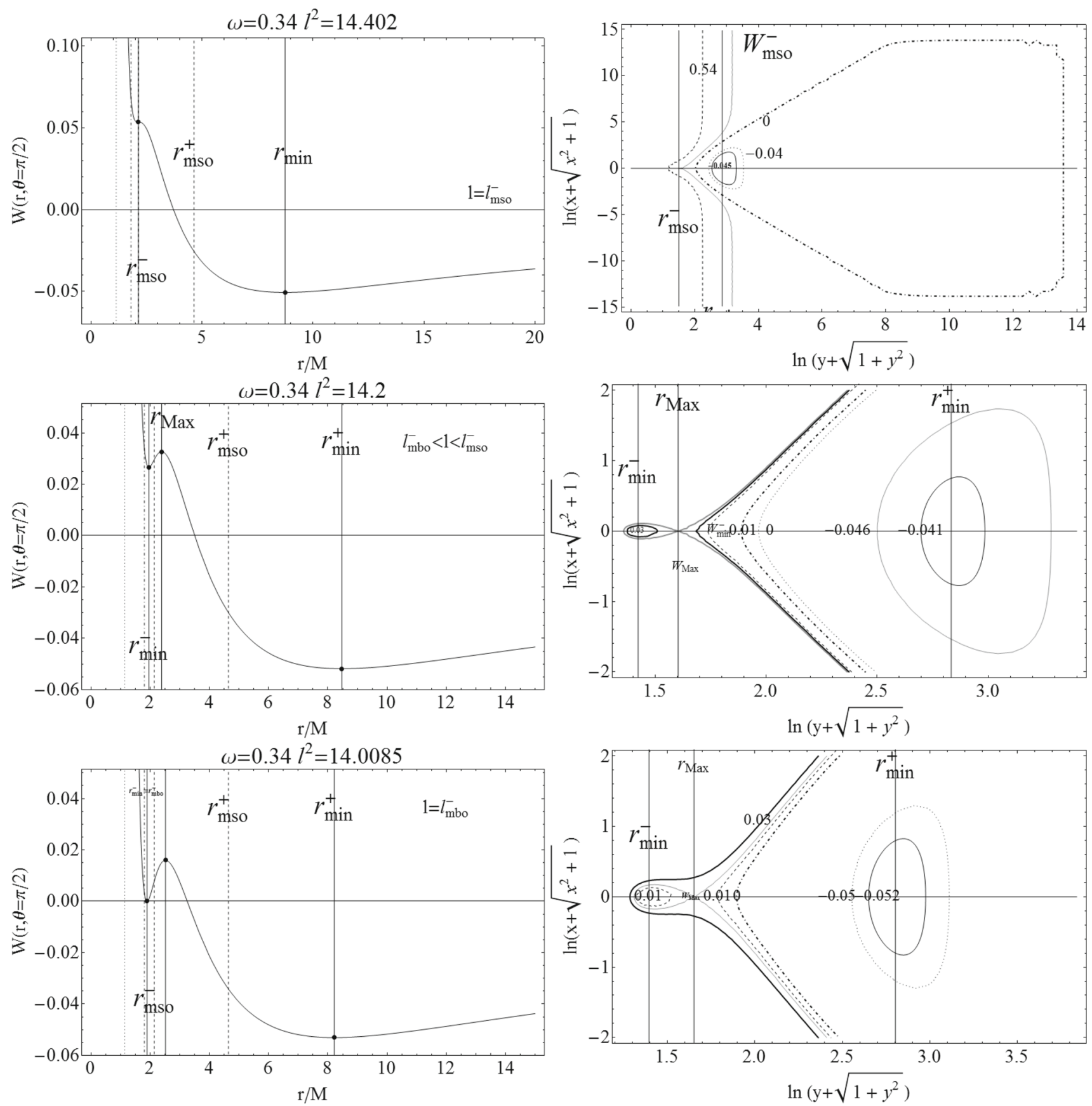

Fig. 7 Region IIIa: $\omega \in] \omega_{m b o}, \omega_{c}[$, naked singularity $\omega=0.34$. Here $\omega M^{2} \rightarrow \omega$. It $l_{m s o}^{-}>l_{m b o}^{-}>l_{m b o}^{+}>l_{\Omega}^{\operatorname{Max}}>l_{m s o}^{+}$, and $r_{\text {stat }}<r_{\Omega}^{\mathrm{Max}}<r_{m b o}^{-}<r_{m s o}^{-}<r_{m b o}^{+}<r_{m s o}^{+}$, where $r / M=$

$\sqrt{x^{2}+y^{2}}$ and $(x, y)$ are Cartesian coordinates. Vertical lines in the right panels set the $r_{i} \in \mathscr{R}$ and the effective potential critical points

This peculiar source separates spacetimes where $r_{\Omega}^{\mathrm{Max}}<$ $r_{m b o}^{-}$(Region III- $a$ ) from those where $r_{\Omega}^{\mathrm{Max}}>r_{m b o}^{-}$(Region III-b). We detail the situation as follows:

$-l>l_{m s o}^{-}$There is only one set of closed configurations with center in $r_{\min } ; W_{\min }<0$. The situation is similar to Fig. 3.

$-l=l_{m s o}^{-}$There is a family of closed $C^{+}$surfaces with center in $r_{\min }^{+}$, and a critical surface with an "inner cusp" located at the saddle point of the $W$ potential where $W_{m s o}^{-}>0$, Fig. 9a. This situation is similar to the configuration at $l=l_{m s o}^{-}$for Hořava parameter in Region IIIa (Fig. 7b).

$-l \in] l_{m b o}^{-}, l_{m s o}^{-}[$This case is illustrated in Fig. 9b. As $\left.W_{\min }^{-} \in\right] 0, W_{\mathrm{Max}}\left[\right.$ and $W_{\min }^{+}<0$, the maximum point 

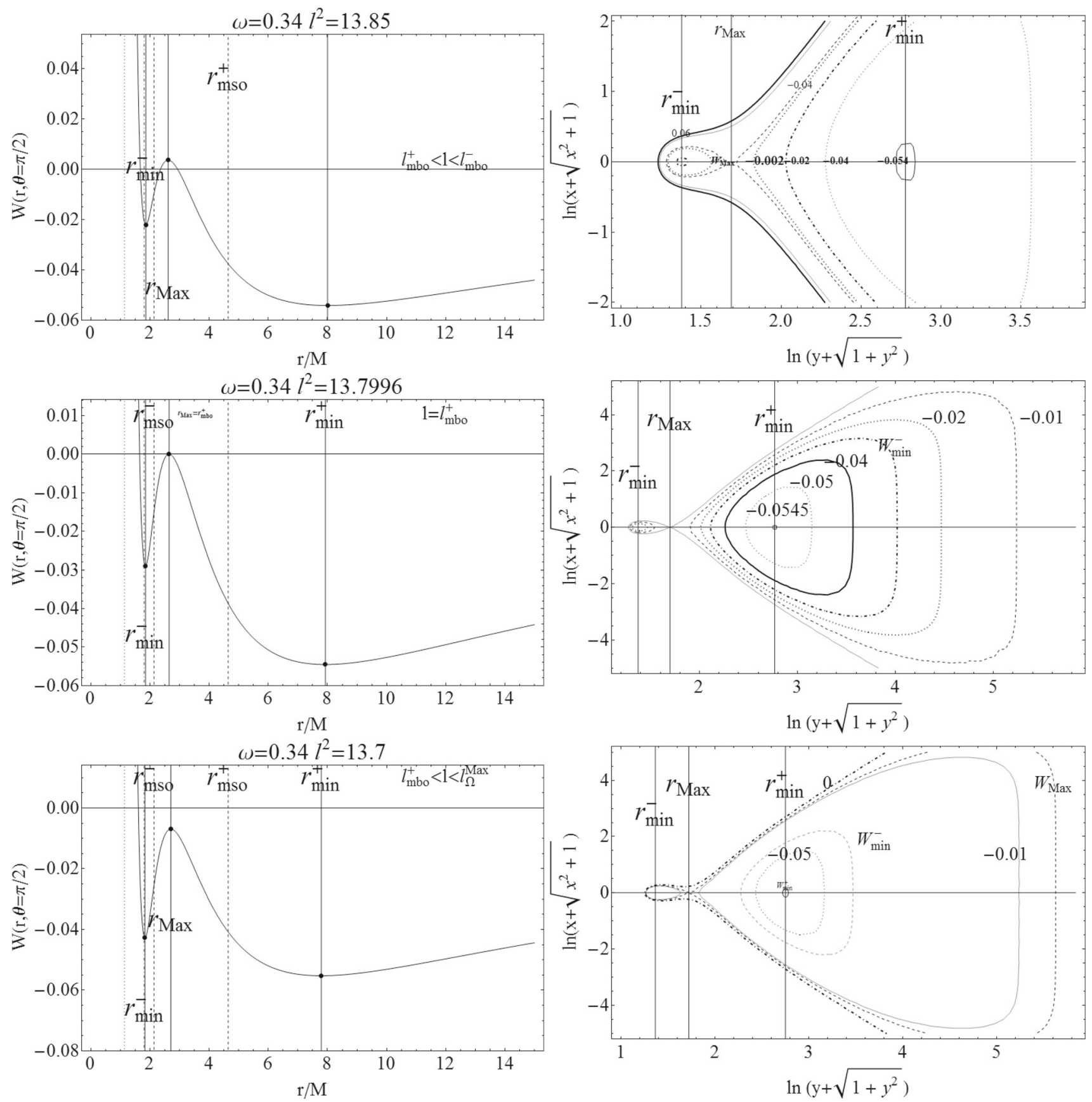

Fig. 8 Region IIIa: $\omega \in] \omega_{m b o}, \omega_{c}$ [ naked singularity $\omega M^{2}=0.34$. Here $\omega M^{2} \rightarrow \omega$. Vertical lines in the right panels set the $r_{i} \in \mathscr{R}$ and the effective potential critical points. We have $l_{m s o}^{-}>l_{m b o}^{-}>l_{m b o}^{+}>$

$r_{\text {Max }}$ gives rise to a critical crossed open surface, with outer cusp associated to matter excretion from the inner configuration to the outer (larger) one. To every inner closed surface centered in $r_{\min }^{-}$an outer open surface is related; see also Fig. 7c.

$-l=l_{m b o}^{-}=l_{\Omega}^{\mathrm{Max}}$ The center, $r_{\Omega}^{\mathrm{Max}}$, of the inner set of closed configurations occurs for $W_{\min }^{-}=0$. The con-

$l_{\Omega}^{\mathrm{Max}}>l_{m s o}^{+}$, and $r_{\mathrm{stat}}<r_{\Omega}^{\mathrm{Max}}<r_{m b o}^{-}<r_{m s o}^{-}<r_{m b o}^{+}<r_{m s o}^{+}$, where $r / M=\sqrt{x^{2}+y^{2}}$ and $(x, y)$ are Cartesian coordinates

figuration structure is shown in Fig. 9c, and it is similar to Fig. 9a.

$-l \in] l_{m b o}^{+}, l_{m b o}^{-}[$Tori illustrated in Fig. 10. There is $W_{\text {Max }}>0, W_{\min }^{-}>W_{\min }^{+}<0$ (class- $I$ configurations). The crossed surface with an outer cusp is opened and can be associated to an excretion configuration. There exist a double class of closed configurations at 

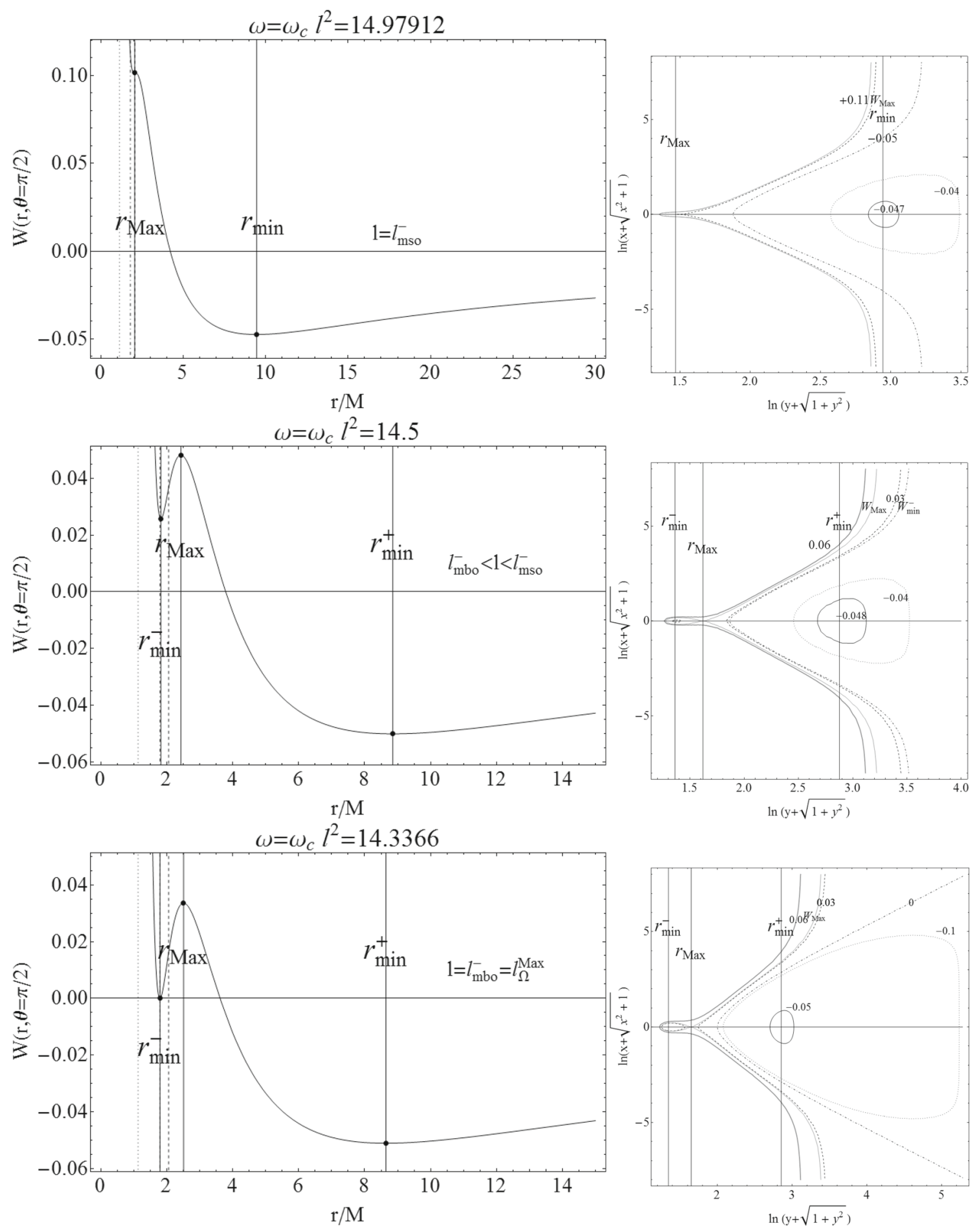

Fig. 9 Naked singularity: $\omega M^{2}=\omega_{c} M^{2}=0.347506$. Here $\omega M^{2} \rightarrow \omega$. We have $l_{m s o}^{-}>l_{\Omega}^{\mathrm{Max}}=l_{m b o}^{-}>l_{m b o}^{+}>l_{m s o}^{+}$, and $r_{\text {stat }}<r_{m b o}^{-}=r_{\Omega}^{\mathrm{Max}}<r_{m s o}^{-}<r_{m b o}^{+}<r_{m s o}^{+}$. We have

$r / M=\sqrt{x^{2}+y^{2}}$ and $(x, y)$ are Cartesian coordinates. Vertical lines in the right panels set the $r_{i} \in \mathscr{R}$ and the effective potential critical points

fixed $W \in] W_{\min }^{-}, 0\left[\right.$. The inner one is centered in $r_{\text {min }}^{-}$ and the outer one in $r_{\min }^{+}$. Closed configurations with $W \in] W_{\min }^{+}, W_{\min }^{-}$[ are centered in $r_{\min }^{+}$(outer disc); a second single set of inner toroidal configuration with center in $r_{\min }^{-}$occurs for $\left.W \in\right] 0, W_{\operatorname{Max}}[$.

$-l=l_{m b o}^{+}$The function $W$ has a saddle point in $r_{m b o}^{+}$where $W_{m b o}^{+}=0$. We have $W_{\min }^{-}<W_{\min }^{+}$(class-III configurations). This situation is similar to the configuration structure of Fig. 6. However, in this last case, $W_{\min }^{-}>W_{\min }^{+}$ (class $I$ ). One could say there is a shift between the 


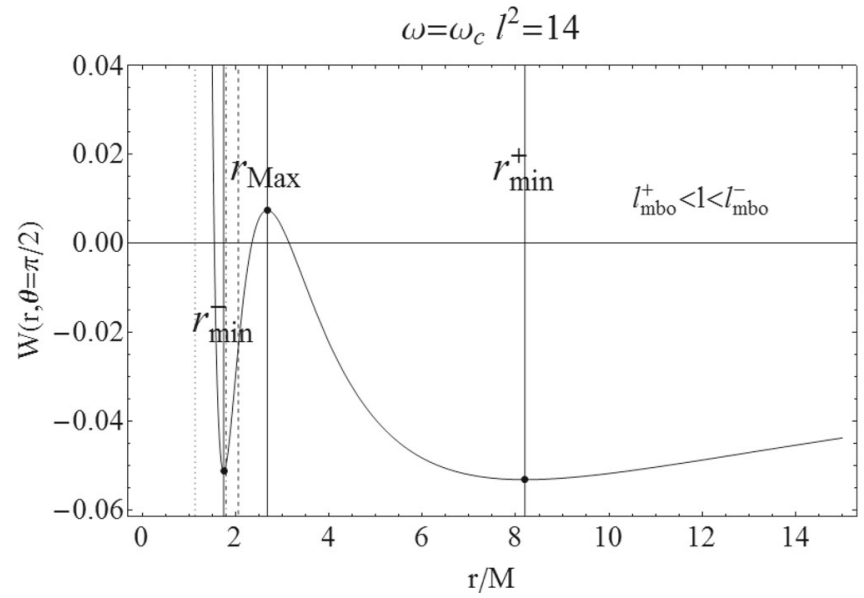

Fig. 10 Naked singularity: $\omega M^{2}=\omega_{c} M^{2}=0.347506$, with $\omega M^{2} \rightarrow \omega$. We have $l_{m s o}^{-}>l_{\Omega}^{\mathrm{Max}}=l_{m b o}^{-}>l_{m b o}^{+}>l_{m s o}^{+}$and $r_{\text {stat }}<r_{m b o}^{-}=r_{\Omega}^{\mathrm{Max}}<r_{m s o}^{-}<r_{m b o}^{+}<r_{m s o}^{+}$. Vertical lines in

class- $I$ and $-I I I$ configurations, with type- $I I$ tori having $W_{\min }^{-}=W_{\min }^{+}<0$.

$-l \in] l_{m s o}^{+}, l_{m b o}^{+}[$The class $I I I$ of configurations is shown in Fig. 10c. As $W_{\text {Max }}<0$, the crossed critical surface is closed and, for $W \in] W_{\mathrm{Max}}, 0[$, there is only one closed surface, with two maximum points for the Boyer surfaces with the centers located on the maximum points for the pressure, $r_{\text {min }}^{ \pm}$; see also Fig. 6 .

$-l=l_{m s o}^{+}$At $r_{m s o}^{+}$there is an outer cusp for the closed configuration with center in $r_{\text {min }}^{-}$. There is only one set of closed surfaces with $W \in] W_{\text {min }}^{-}, 0[$. We can consider two subclasses of configurations: one, belonging to $W \in] W_{\min }^{-}, W_{m s o}^{+}$, centered in $r_{\min }^{-}$, with outer edge in $r \in] r_{\min }, r_{m s o}^{+}[$, and the second one belonging to $W \in] W_{m s o}^{+}$, 0[. The maximum of pressure for these configurations is still located in $r_{\text {min }}^{-}$, but the outer edges of the discs are at $r>r_{m s o}^{+}$. Similar configurations occur in Fig. 5.

$-l \in] l_{\text {stat }}, l_{m s o}^{+}[$There is one set of closed configurations with center in $r_{\min }^{-}$, see Fig. 3.

\subsubsection{Region IIIb: $\omega \in] \omega_{c}, \omega_{\gamma}[$}

In the naked singularity spacetimes with Hořava parameter in $\omega \in] \omega_{c}, \omega_{\gamma}$ [, the following relations are fulfilled: $r_{\text {stat }}<r_{m b o}^{-}<r_{\Omega}^{\mathrm{Max}}<r_{m s o}^{-}<r_{m b o}^{+}<r_{m s o}^{+}$and $l_{m s o}^{-}>l_{\Omega}^{\mathrm{Max}}>l_{\text {mbo }}^{-}>l_{\text {mbo }}^{+}>l_{\text {mso }}^{+}$. Region IIIb is a part of Class-II attractors considered in Sect. 3.3.2. As in Region $I I I-a$, this set of sources is characterized by excretion. We detail the situation as follows:

$-l>l_{m s o}^{-}$There is one set of closed configurations with center in $r_{\min } ; W_{\min }<0-$ see Fig. 3.

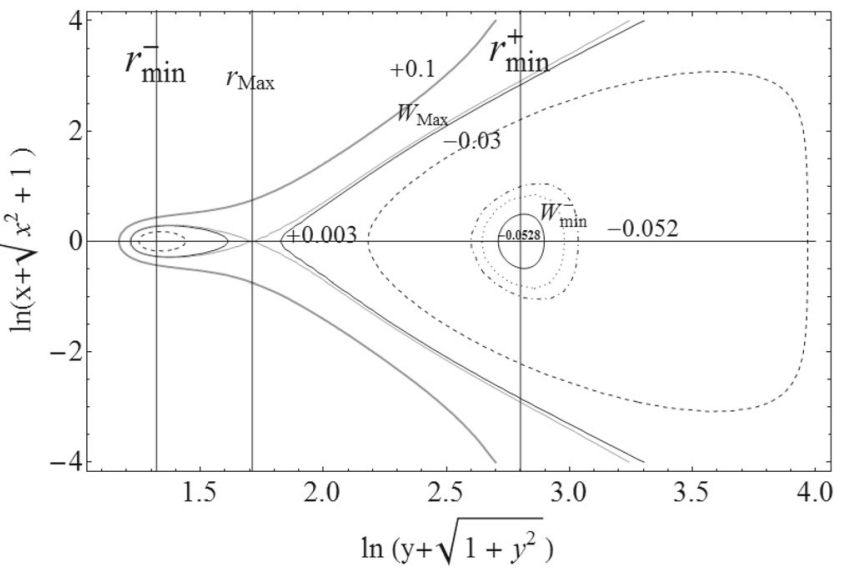

the right panels set the $r_{i} \in \mathscr{R}$ and the effective potential critical points. $r / M=\sqrt{x^{2}+y^{2}}$ and $(x, y)$ are Cartesian coordinates

$-l=l_{m s o}^{-}$A saddle point of $W$ is at $r_{m s o}^{-}$. Since $W_{m s o}^{-}>$ 0 there is a set of closed configuration centered in the minimum $r_{\min }$ and an open configuration with inner cusp. The two open branches are aligned on the axis (this is therefore a discriminant case); see Fig. 11.

$-l \in] l_{\Omega}^{\mathrm{Max}}, l_{m s o}^{-}$[ There are two minima of the $W$ function: $W_{\min }^{-}>0$ and $W_{\min }^{+}<0$. Consequently a doubled configuration $C^{ \pm}$of type $I$ is possible, at different $K$ centered, respectively, in $r_{\min }^{-}$, the inner one $C^{-}$; in $r_{\min }^{+}$, the outer one $C^{+}$. The maximum corresponds to an open crossed surface, with an outer cusp. This case is illustrated in Fig. 11b; see also Fig. 9a.

$-l=l_{\Omega}^{\mathrm{Max}}$ The center of the inner disc $C^{-}$is located in $r_{\Omega}^{\mathrm{Max}}$, where $W_{\Omega}^{\mathrm{Max}}>0$. This case is illustrated in Fig. 11c and corresponds to the type- $I$ configuration.

$-l \in] l_{m b o}^{-}, l_{\Omega}^{\mathrm{Max}}[$ There is an excretion open crossed $I$ configuration; see Fig. 12a.

$-l=l_{m b o}^{-}$The center of the inner $I$ disc is at $W_{m b o}^{-}$. This situation is similar to the case $l \in] l_{\Omega}^{\mathrm{Max}}, l_{m b o}^{-}[$and is sketched in Fig. 6.

$-l \in] l_{m b o}^{+}, l_{m b o}^{-}$[ These configurations of type $I I I$ is shown in Fig. 12c. Two configurations $C^{ \pm}$are therefore possible at equal $W \in] W_{\min }^{+}, 0[$. Excretion towards the exterior is possible. In general there is a sequence of $I-I I-I I I$ configurations.

$-l=l_{m b o}^{+}$Respect to the case $\left.l \in\right] l_{m b o}^{+}, l_{m b o}^{-}[$the maximum point is at $r_{m b o}^{+}$, where $W_{m b o}^{+}=0$. There is a crossed open III configuration, Fig. 12d.

$-l \in] l_{m s o}^{+}, l_{m b o}^{+}[$There is a crossed III closed surface; see Fig. 6.

$-l=l_{m s o}^{+}$There is an outer "cusp" in $r_{m s o}^{+}$, see Fig. 5.

$-l \in] l_{\text {stat }}, l_{m s o}^{+}$[ Only closed toroidal surfaces with center in $r_{\text {min }}$ are possible, Fig. 13. 

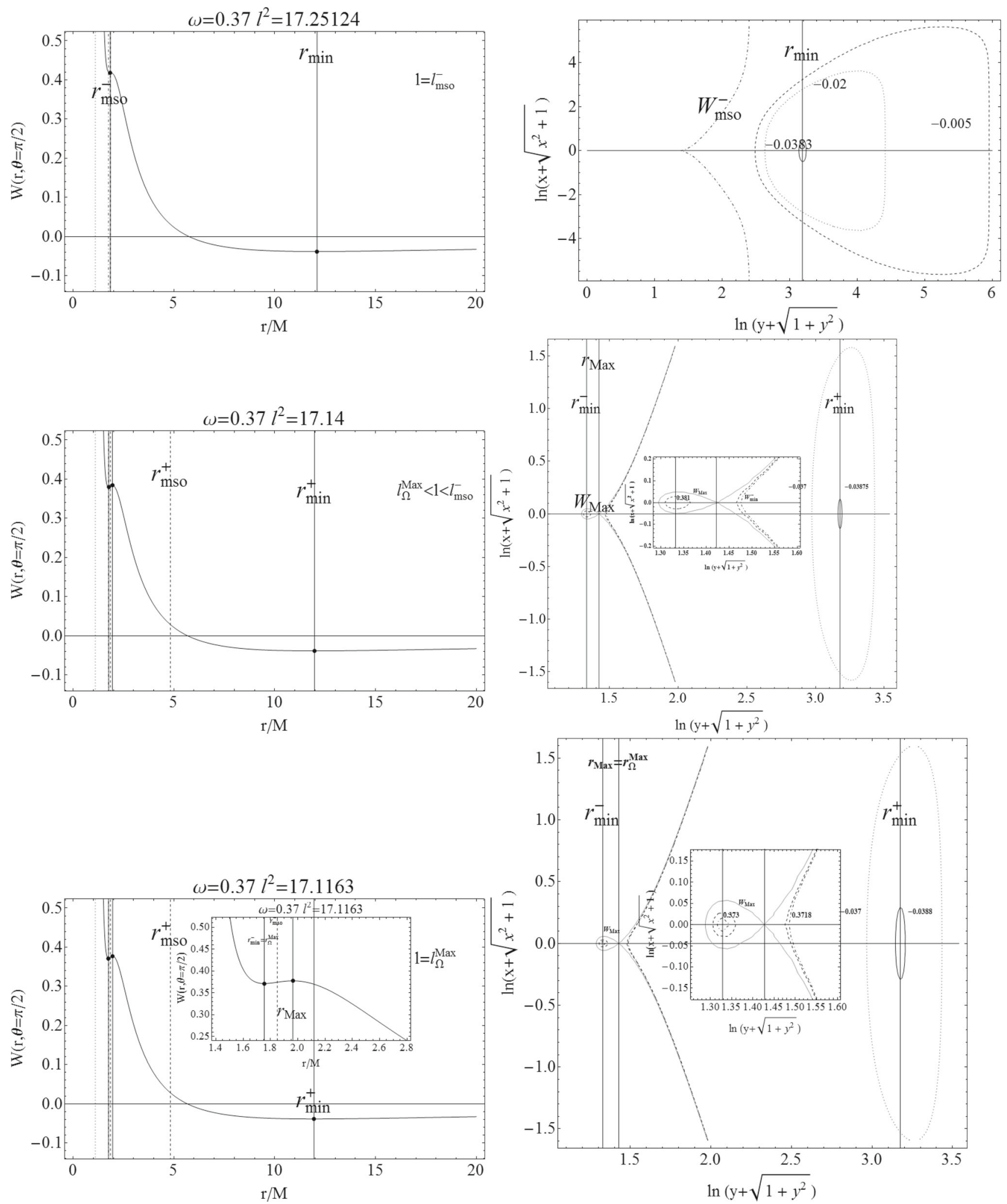

Fig. 11 Region IIIb: $\omega \in] \omega_{c}, \omega_{\gamma}\left[\right.$, naked singularity $\omega M^{2}=0.37$, where $\omega M^{2} \rightarrow \omega$. We have $l_{m s o}^{-}>l_{\Omega}^{\mathrm{Max}}>l_{m b o}^{-}>l_{m b o}^{+}>$ $l_{m s o}^{+}$, and $r_{\text {stat }}<r_{m b o}^{-}<r_{\Omega}^{\mathrm{Max}}<r_{m s o}^{-}<r_{m b o}^{+}<r_{m s o}^{+}$. Ver-

tical lines in the right panels set the $r_{i} \in \mathscr{R}$ and the effective potential critical points. $r / M=\sqrt{x^{2}+y^{2}}$ and $(x, y)$ are Cartesian coordinates 

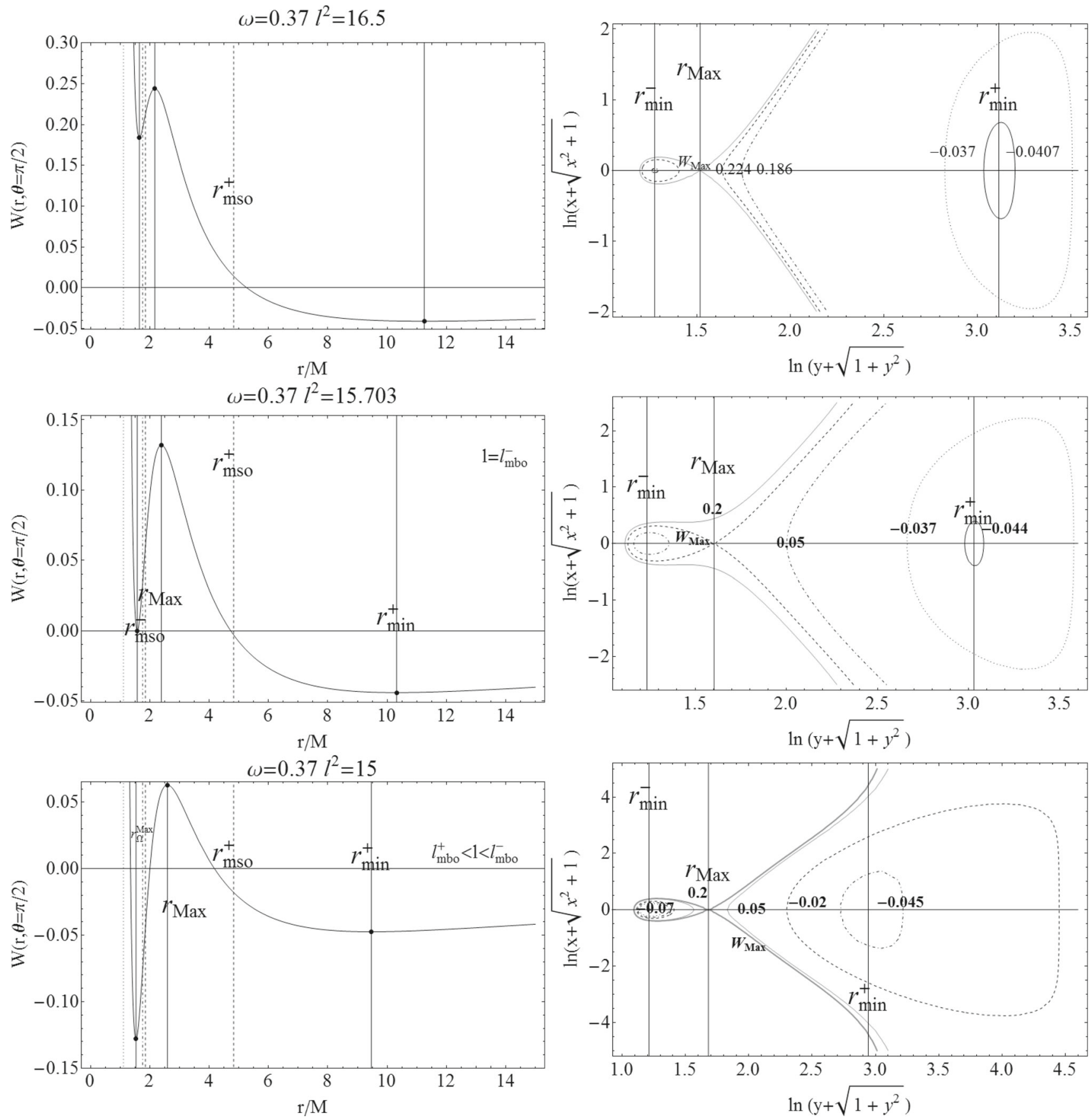

Fig. 12 Region IIIb: $\omega \in] \omega_{c}, \omega_{\gamma}$, naked singularity $\omega M^{2}=0.37$, where $\omega M^{2} \rightarrow \omega$, we have $l_{m s o}^{-}>l_{\Omega}^{\text {Max }}>l_{m b o}^{-}>l_{m b o}^{+}>l_{m s o}^{+}$, and $r_{\text {stat }}<r_{m b o}^{-}<r_{\Omega}^{\mathrm{Max}}<r_{m s o}^{-}<r_{m b o}^{+}<r_{m s o}^{+}$. Vertical lines in the right panels set the $r_{i} \in \mathscr{R}$ and the effective potential critical points

\subsubsection{Naked singularity: $\omega=\omega_{\gamma}$}

We analyze properties of the fluid tori in the special $\mathrm{K}-\mathrm{S}$ spacetime with $\omega=\omega_{\gamma}$ where $r_{\text {stat }}<r_{m b o}^{-}<r_{\Omega}^{\operatorname{Max}}=$ $r_{\gamma}^{ \pm}=r_{m s o}^{-}<r_{m b o}^{+}<r_{m s o}^{+}$and $l_{m s o}^{-}=l_{\Omega}^{\mathrm{Max}}>l_{m b o}^{-}>$ $l_{m b o}^{+}>l_{m s o}^{+}$. This is a discriminant case splitting the $\mathrm{K}-\mathrm{S}$ naked singularity spacetimes containing two photon circular orbits $\omega \in] \omega_{\gamma}, 1 / 2[$, from the sources in the Regions $I-I I-$ IIIa-IIIb with no photon orbits.

$-l \geq l_{m s o}^{-}=l_{\Omega}^{\mathrm{Max}}$ There are only closed configurations; see Fig. 3.

$-l \in\left[l_{m b o}^{-}, l_{\Omega}^{\mathrm{Max}}\right.$ [ This case is illustrated in Figs. 14 and 15 . There is a closed $C^{+}$configuration centered in $r_{\min }^{+}$and 


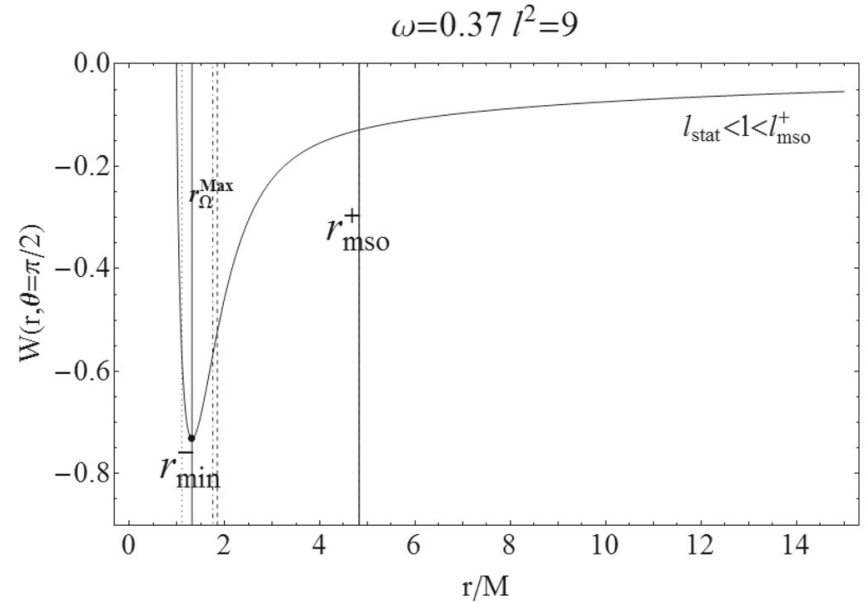

Fig. 13 Region IIIb: $\omega \in] \omega_{c}, \omega_{\gamma}\left[\right.$, naked singularity $\omega M^{2}=0.37$, where $\omega M^{2} \rightarrow \omega$. We have $l_{m s o}^{-}>l_{\Omega}^{\mathrm{Max}}>l_{m b o}^{-}>l_{m b o}^{+}>l_{m s o}^{+}$, and $r_{\text {stat }}<r_{m b o}^{-}<r_{\Omega}^{\mathrm{Max}}<r_{m s o}^{-}<r_{m b o}^{+}<r_{m s o}^{+} \cdot r / M=$

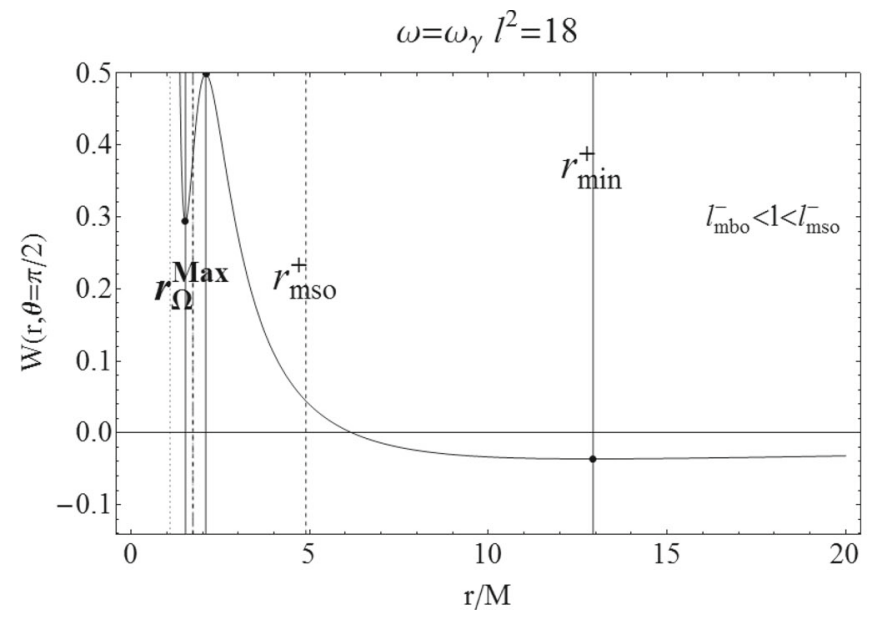

Fig. 14 Naked singularity: $\omega=\omega_{\gamma}$. Here $\omega M^{2} \rightarrow \omega$. We have $l_{m s o}^{-}=l_{\Omega}^{\mathrm{Max}}>l_{m b o}^{-}>l_{m b o}^{+}>l_{m s o}^{+}$, and $r_{\text {stat }}<$ $r_{m b o}^{-}<r_{\Omega}^{\operatorname{Max}}=r_{\gamma}^{ \pm}=r_{m s o}^{-}<r_{m b o}^{+}<r_{m s o}^{+} \cdot r / M=$

an inner $C^{-}$one with center in $r_{\min }^{-}$, having an open excretion point. This is a type- $I$ configuration.

- $\left.l \in] l_{m b o}^{+}, l_{m b o}^{-}\right]$This case of the class-III configurations is illustrated in Fig. 15. There is a shift between a $I$ and $I I I$ configuration, where a type- $I I$ case with $W_{\min }^{-}=W_{\min }^{+}<$ 0 appears.

$-l \in] l_{m s o}^{+}, l_{m b o}^{+}\left[\right.$There are double closed $C^{ \pm} I I I$ configurations eventually closing into a single closed crossed configuration; see Fig. 6.

$-l=l_{m s o}^{+}$An outer cusp is located in $r_{m s o}^{+}$. There is only one class of closed $C^{-}$excretion configurations - see Fig. 5.

$-l \in] l_{\text {stat }}, l_{m s o}^{+}[$Only closed toroidal surfaces are possible, see Fig. 3.

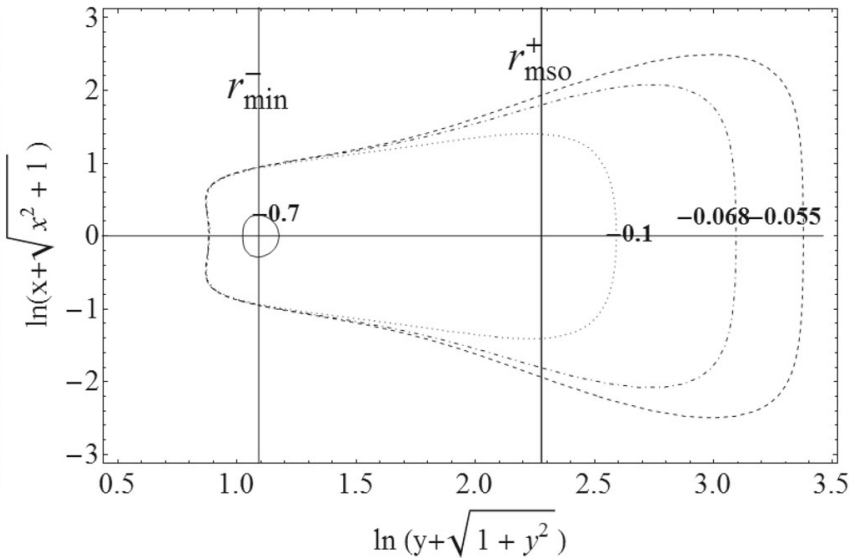

$\sqrt{x^{2}+y^{2}}$ and $(x, y)$ are Cartesian coordinates. Vertical lines in the right panels set the $r_{i} \in \mathscr{R}$ and the effective potential critical points

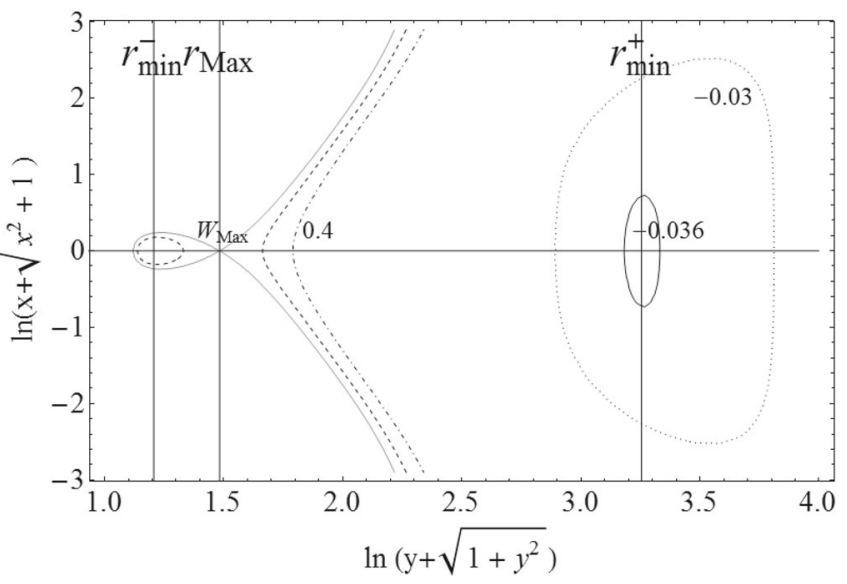

$\sqrt{x^{2}+y^{2}}$ and $(x, y)$ are Cartesian coordinates. Vertical lines in the right panels set the $r_{i} \in \mathscr{R}$ and the effective potential critical points

\subsubsection{Region IV: $\omega \in] \omega_{\gamma}, 0.5[$}

Kehagias-Sfetsos naked singularity spacetimes in Region IV contain two photon circular orbits, the inner a stable one at $r_{\gamma}^{-}$, the outer an unstable one at $r_{\gamma}^{+}$, and the stable circular orbit $r_{m s o}^{+}$. We have $r_{\text {stat }}<r_{m b o}^{-}<r_{\gamma}^{-}<r_{\gamma}^{+}<r_{m b o}^{+}<r_{m s o}^{+}$ and $l_{\gamma}^{-}>l_{\gamma}^{+}>l_{m b o}^{-}>l_{m b o}^{+}>l_{m s o}^{+}$. This region of attractors corresponds to the Class III detailed in Sect. 3.3.3). In the curve $\left.\Delta_{\gamma}^{ \pm} \equiv\right] r_{\gamma}^{-}, r_{\gamma}^{+}$[, no circular geodesics are allowed, i.e., there is no critical point of the effective potential and, therefore, no disc center or accretion/excretion point or cusp can be located in $\Delta_{\gamma}^{ \pm}$. Then there is no critical point for the angular frequency $\Omega$. 


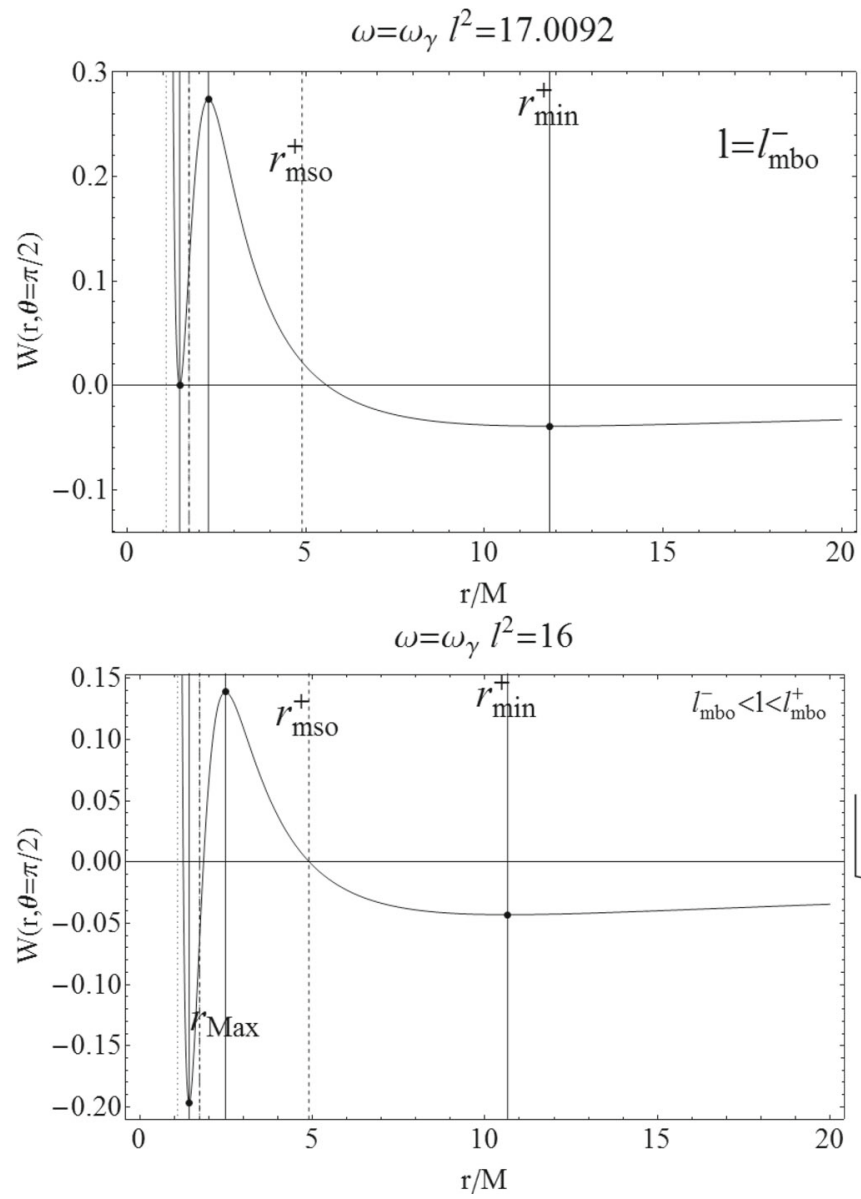

Fig. 15 Naked singularity $\omega=\omega_{\gamma}$. Here $\omega M^{2} \rightarrow \omega$. Vertical lines in the right panels set the $r_{i} \in \mathscr{R}$ and the effective potential critical points. We have $l_{m s o}^{-}=l_{\Omega}^{\mathrm{Max}}>l_{m b o}^{-}>l_{m b o}^{+}>l_{m s o}^{+}$, and $r_{\text {stat }}<r_{m b o}^{-}<$

$-l \geq l_{\gamma}^{-}$This situation is sketched in Fig. 3: there is one class of closed configurations.

$\left.-l \in] l_{\gamma}^{+}, l_{\gamma}^{-}\right]$There is a minimum of the effective potential in $r_{\text {min }}^{+}$with $W_{\text {min }}^{+}<0$, giving the center of a set of closed $C^{+}$toroidal surfaces. The effective potential has a further critical point in $r_{\text {min }}^{-}<r_{\gamma}^{-}$, with $W_{\text {min }}^{-}>0$. Correspondingly, there is a sequence of inner (very narrow) $C^{-}$discs centered in $r_{\min }^{-}$. However, two sets of closed configurations are separated by means of the open surface corresponding to the second solution for $W=$ const : $W \in\left[W_{\min }^{-},+\infty[\right.$ slightly "cusped" from the interior toward the exterior solution and aligned along the axis; see Fig. 16. This is a type- $I$ configuration.

$-l \in] l_{m b o}^{-}, l_{\gamma}^{+}[$This situation is illustrated in Fig. 17a. As $W_{\min }^{-}>0>W_{\min }^{+}$(class $I$ ), we have $r_{\text {Max }}>r_{\gamma}^{+}$, and a crossed open surface, with exterior cusp (excretion configuration), with branches aligned to the axis, separated from the inner closed discs (with $W>0$ ) and, the disc center occurs in $r_{\text {min }}^{+}$.
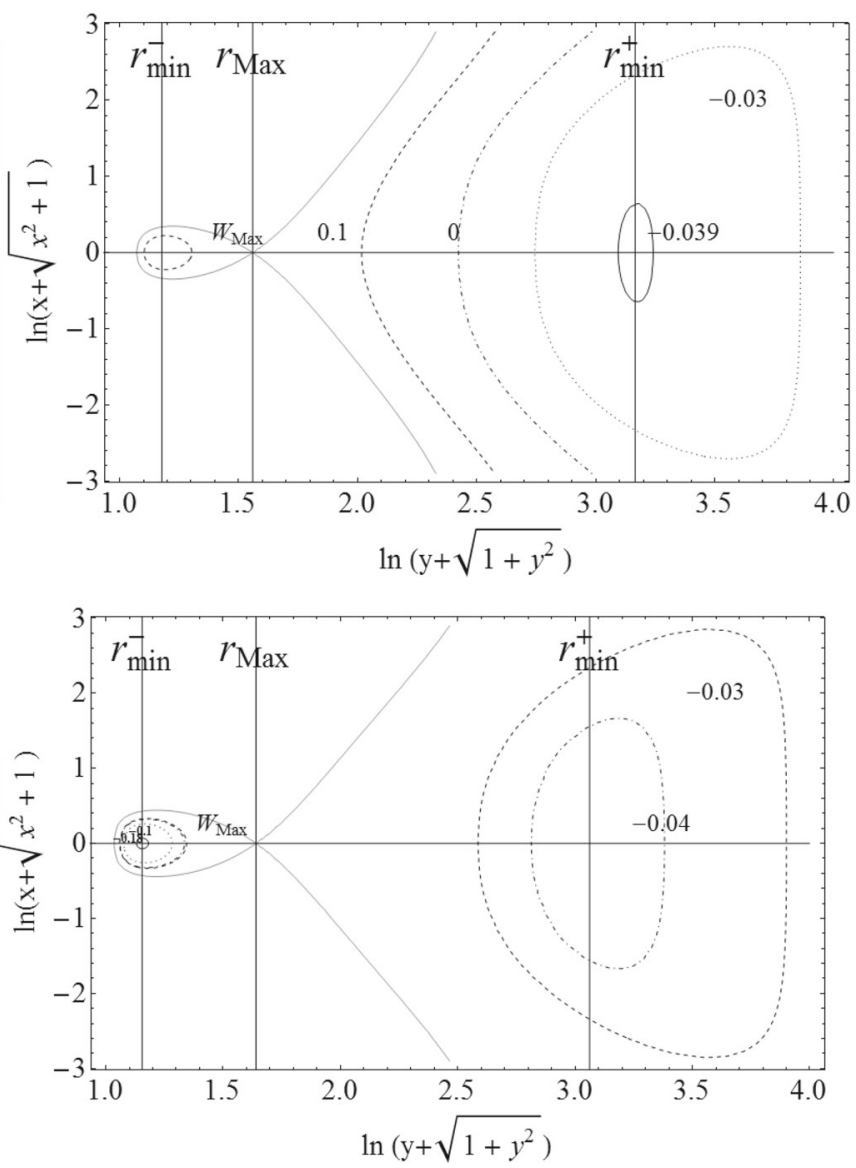

$r_{\Omega}^{\mathrm{Max}}=r_{\gamma}^{ \pm}=r_{m s o}^{-}<r_{m b o}^{+}<r_{m s o}^{+} . r / M=\sqrt{x^{2}+y^{2}}$ and $(x, y)$ are Cartesian coordinates

$-l=l_{m b o}^{-}$This case is shown in Fig. 17b, the minimum of $W$ is located in $r_{m b o}^{-}$, with $W_{\text {min }}^{-}=0$, and the situation is similar to configurations at $l \in] l_{m b o}^{+}, l_{\gamma}^{+}[$in Fig. 17a.

$-l \in] l_{m b o}^{+}, l_{m b o}^{-}$[ This class-III configuration is shown in Fig. 17c. There are two sets $C^{ \pm}$of closed configurations separated by an open crossed excretion surface.

$-l=l_{m b o}^{+}$The maximum of $W$ is at $r_{m b o}^{+}$. This corresponds to an open crossed excretion surface; see Fig. 6.

$-l \in] l_{m b o}^{-}, l_{m s o}^{+}\left[\right.$The critical points are at $W_{\text {crit }}<0$. There are closed surfaces ranged by the closed crossed one as in Fig. 6.

$-l=l_{m s o}^{+}$There is an outer cusp for $W_{m s o}^{+}$, see Fig. 5 .

$-l<l_{m s o}^{+}$There are closed crossed surfaces centered in $r_{\text {min }}^{+}$; see Fig. 3c, d.

6.2 Extreme case: $\omega M^{2}=\omega_{h} M^{2}=0.5$

In this section we consider the extreme Kehagias-Sfetsos BH spacetime: $\omega M^{2}=0.5$. We have $r_{ \pm}=M, r_{ \pm}<r_{\gamma}^{+}<$ 

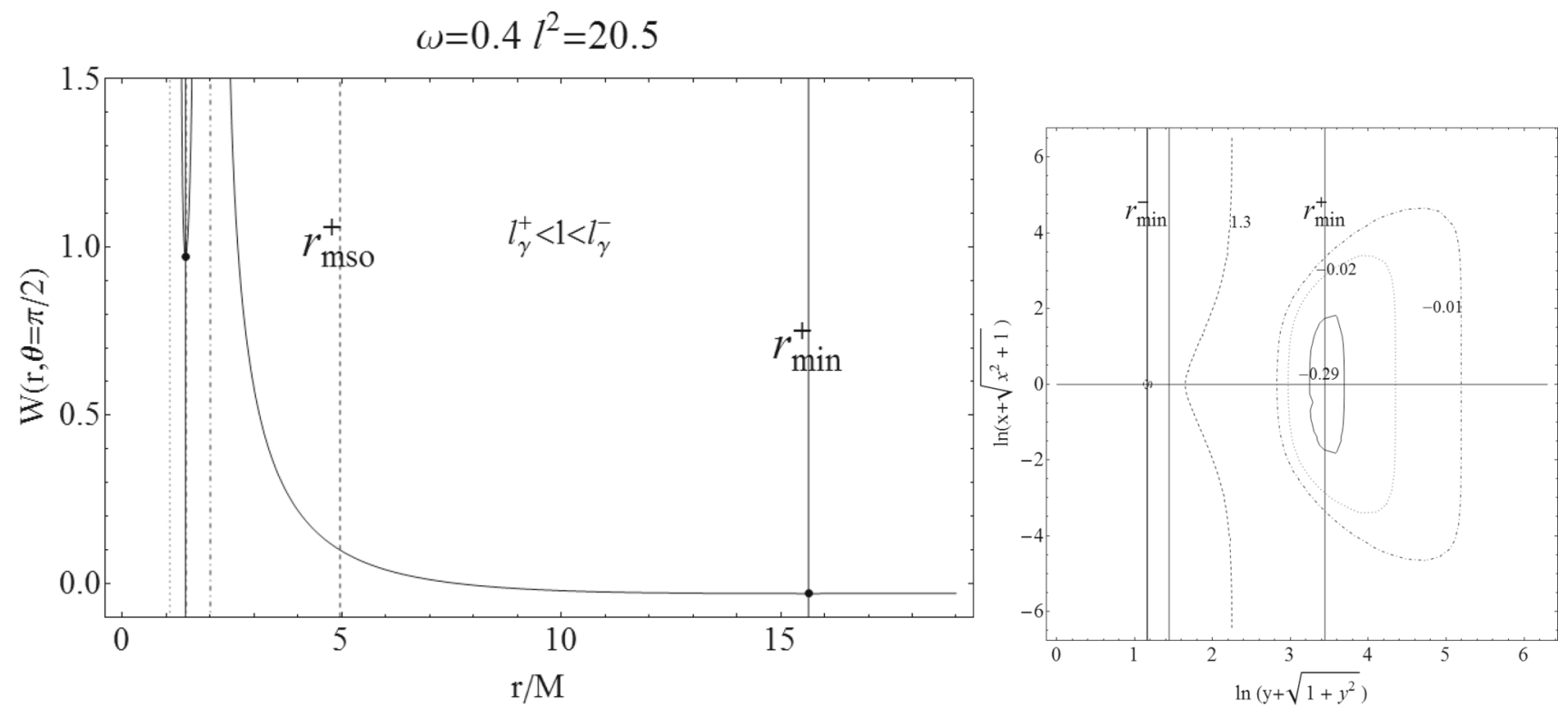

Fig. 16 Region IV: $\omega \in] \omega_{\gamma}, 0.5\left[\right.$. Here $\omega M^{2} \rightarrow \omega$. We have $\omega M^{2}=$ 0.4 with $l_{\gamma}^{-}>l_{\gamma}^{+}>l_{m b o}^{-}>l_{m b o}^{+}>l_{m s o}^{+}$, and $r_{\text {stat }}<r_{m b o}^{-}<r_{\gamma}^{-}<$ $r_{\gamma}^{+}<r_{m b o}^{+}<r_{m s o}^{+} . r / M=\sqrt{x^{2}+y^{2}}$ and $(x, y)$ are Cartesian coordi-

$r_{m b o}^{+}<r_{m s o}^{+}$, and $l_{\gamma}^{+}>l_{m b o}^{+}>l_{m s o}^{+}$. The circular orbits arrangement has been described in Sect. 3.3.4. There is only one photon orbit located in $r_{\gamma}^{+}$, which is the inner limit for circular orbits or the cusp configurations.

$-l \geq l_{\gamma}^{+}$There is a set of closed configuration and correspondingly a set of inner ones, embracing the black hole; see Fig. 18a.

$-l \in] l_{m b o}^{+}, l_{\gamma}^{+}[$There are open configurations with a cross point and a closed set of outer ones - see Fig. 18c.

$-l=l_{m b o}^{+}$This is a critical case where the maximum $W_{\text {Max }}=0$ is located in $r_{m b o}^{+}-$Fig. $18 \mathrm{~d}$.

$-l \in] l_{m s o}^{+}, l_{m b o}^{+}[$There is one maximum of the potential, $W_{\text {Max }}<0$, and a minimum, $W_{\min }<0$. The inner and outer configurations are connected by the crossed closed surface; see Fig. 19a

$-l=l_{m s o}^{+}$These is a saddle point of $W$ located in $r_{m s o}^{+}$. Consequently, there is an outer cusped surface for the inner set of surfaces; see Fig. 19b.

$-l<l_{m s o}^{+}$There are only inner surfaces as in Fig. 19c.

6.3 The black hole case $\omega M^{2}>0.5$

We consider the perfect fluid orbiting the Kehagias-Sfetsos black holes in the region $r>r_{+}$. The properties of the circular motion were discussed in Sect. 3.3.4. The structure of the Boyer surfaces is similar to the extreme-BH case analyzed in Sect. 6.2, and it can be summarized as follows: nates. Left panels dotted-dashed lines are the radii $r_{\gamma}^{-}<r_{\gamma}^{+}$, a dotted line is $r_{\text {stat }}$. Vertical lines in the right panels set the $r_{i} \in \mathscr{R}$ and the effective potential critical points

$-l \geq l_{\gamma}^{+}$This case is illustrated in Fig. 20a, there is a set of outer closed surfaces and an inner one. No cusped, critical, surfaces are possible.

$-l \in\left[l_{m b o}^{+}, l_{\gamma}^{+}[\right.$There is a set of outer closed surfaces and the inner ones, a crossed open one is for $W_{\text {Max }}$; see Figs. 20b, c.

$\left.-l \in] l_{m s o}^{+}, l_{m b o}^{+}\right]$There is a set of outer closed surfaces and inner ones, a crossed one is for $W=W_{\text {Max }}$; see Fig. 21a.

$-l=l_{m s o}^{+}$The outer cusp appears for the inner closed surfaces; see Fig. 21b.

$-l<l_{m s o}^{+}$There is no critical point for the effective potential and the configurations are as in Fig. 21c.

\section{Discussion and conclusions}

We analyzed the structure and shape of perfect fluid toroidal equilibrium tori orbiting the Kehagias-Sfetsos (K-S) sources. We considered the tori for the whole range of the dimensionless parameter $\omega M^{2}<1 / 2$ corresponding to naked singularity spacetimes and the values $\omega M^{2} \geq 1 / 2$ for the black hole spacetimes. From the characterization of the toroidal configurations we create a classification of $\mathrm{K}-$ $\mathrm{S}$ spacetimes having peculiar and distinctive features with respect to the matter dynamics. According to the properties of the orbiting fluids we are able to distinguish four classes of naked singularity sources corresponding to four regions of values of the quantum parameter $\omega M^{2}$. The extreme $\mathrm{BH}$ case $\left(\omega M^{2}=1 / 2\right)$ is addressed in Sect. 6.2 and the geometries 

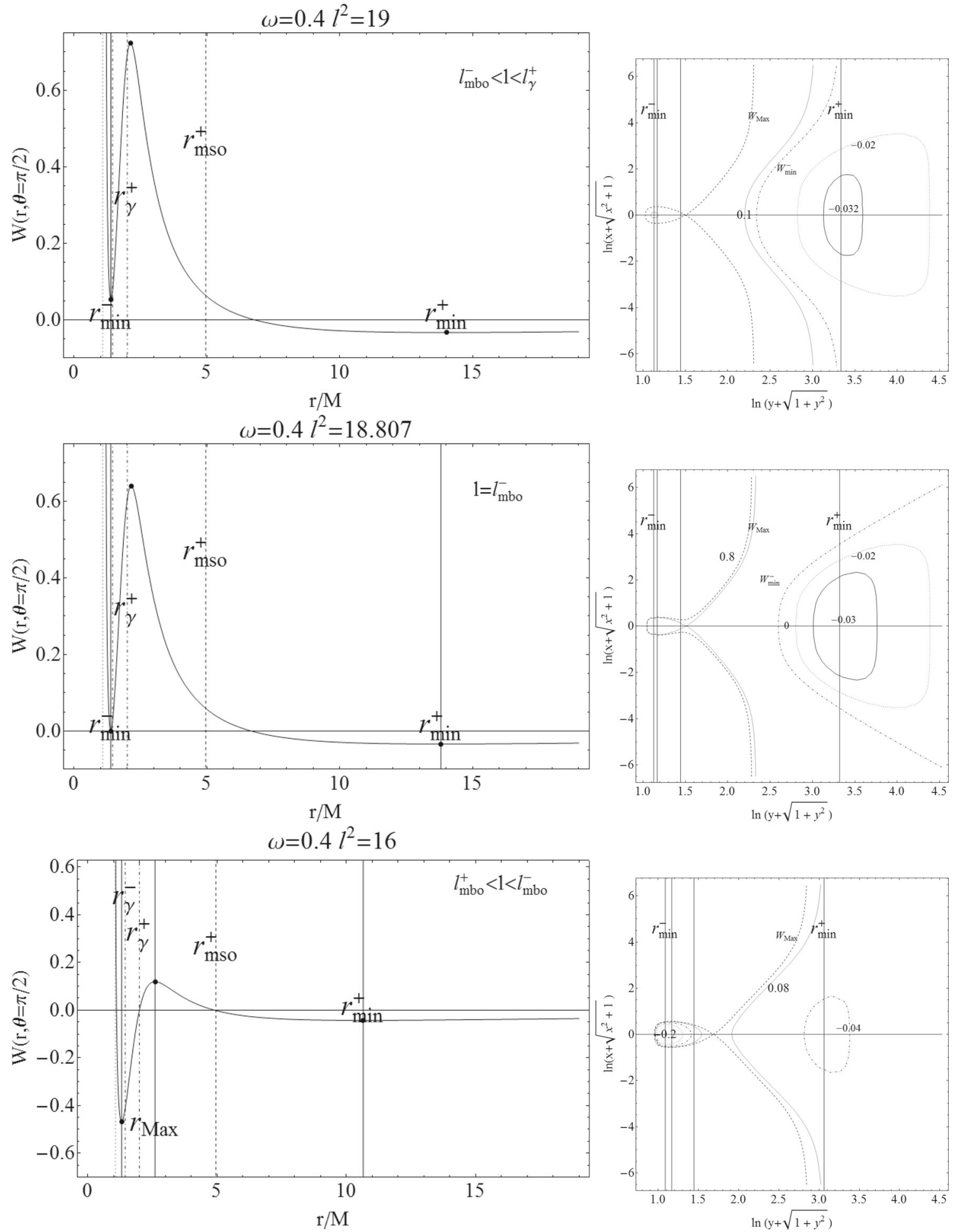

Fig. 17 Region IV: $\omega \in] \omega_{\gamma}, 0.5\left[\right.$. We have $\omega M^{2}=0.4$ with $l_{\gamma}^{-}>$ $l_{\gamma}^{+}>l_{m b o}^{-}>l_{m b o}^{+}>l_{m s o}^{+}$, and $r_{\text {stat }}<r_{m b o}^{-}<r_{\gamma}^{-}<r_{\gamma}^{+}<r_{m b o}^{+}<$ $r_{m s o}^{+}$. Here $\omega M^{2} \rightarrow \omega \cdot r / M=\sqrt{x^{2}+y^{2}}$ and $(x, y)$ are Cartesian

coordinates. Vertical lines in the right panels set the $r_{i} \in \mathscr{R}$ and the effective potential critical points. Left panels dotted-dashed lines are the radii $r_{\gamma}^{-}<r_{\gamma}^{+}$, a dotted line is $r_{\text {stat }}$

defined with the limiting values of the quantum parameter in $C_{i}$ with respect to the stability and morphology of the configurations were explored in detail.

The first class of naked singularities, identified as Region $I$ with $\omega \in] 0, \omega_{m s o}[$, is studied in Sect. 6.1.1: only stable circular orbits are allowed. Geometries belonging to 

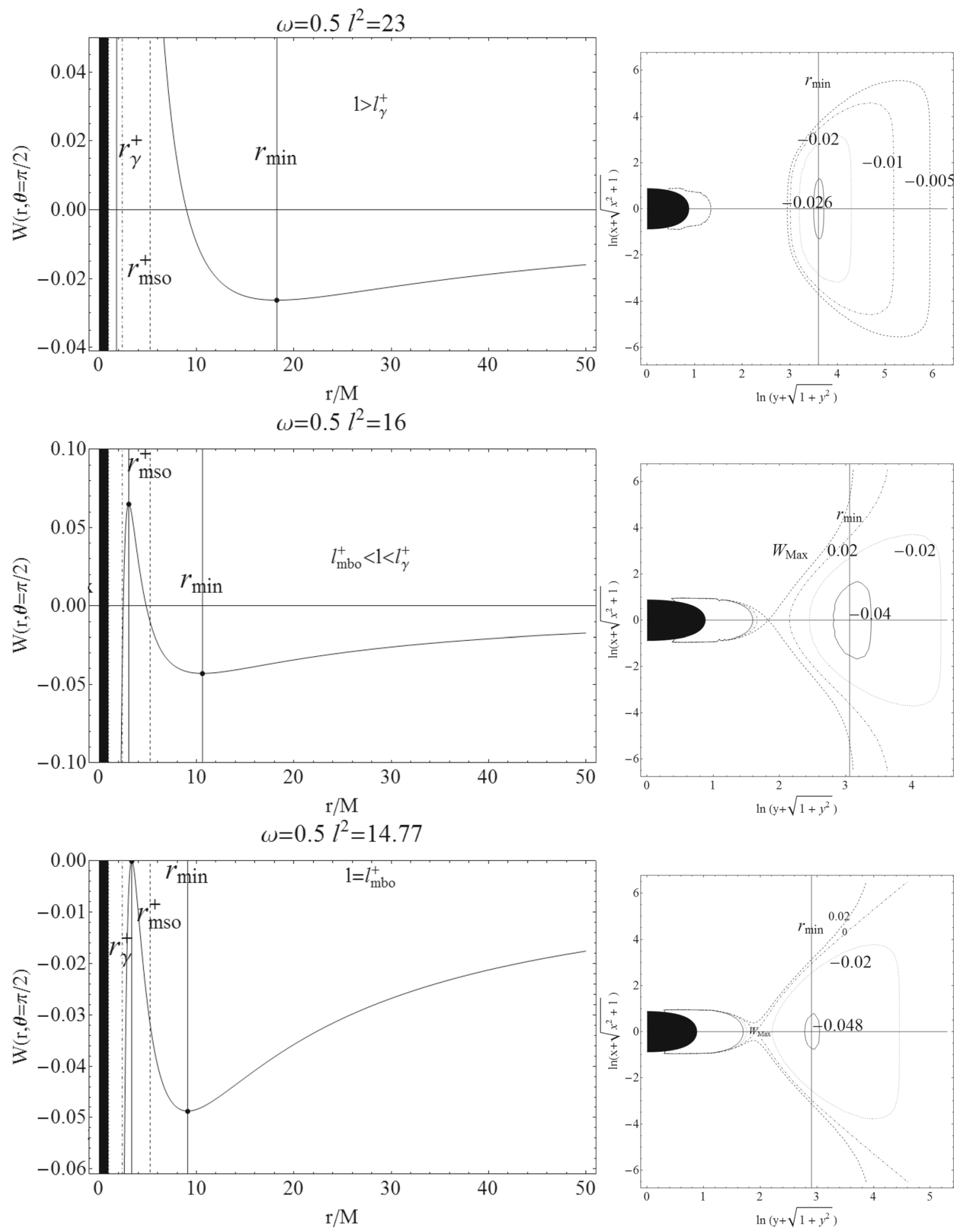

Fig. 18 Extreme black hole case: $\omega M^{2}=0.5$. We have $r_{ \pm}=M$, $r_{ \pm}<r_{\gamma}^{+}<r_{m b o}^{+}<r_{m s o}^{+}$, and $l_{\gamma}^{+}>l_{m b o}^{+}>l_{m s o}^{+}$. The black region is $r<r_{+}$. Here $\omega M^{2} \rightarrow \omega . r / M=\sqrt{x^{2}+y^{2}}$ and $(x, y)$ are Carte-

Region II with $\omega \in] \omega_{m s o}, \omega_{m b o}$ [, as detailed in Sect. 6.1.3, are characterized by two marginally stable circular orbits; this case is further articulated as it contains two regions of orbital stability separated by an instability region. Region sian coordinates. Vertical lines in the right panels set the $r_{i} \in \mathscr{R}$ and the effective potential critical points. The black region is $r<r_{+}$. The dotted-dashed line in the left panels is $r_{\gamma}^{ \pm}$

III splits into two sets: Region IIIa with $\omega \in] \omega_{m b o}, \omega_{c}[$, considered in Sect. 6.1.5, presents two marginally bounded orbits and the situation is as follows: $r_{\text {stat }}<r_{\Omega}^{\mathrm{Max}}<r_{m b o}^{-}<$ $r_{m s o}^{-}<r_{m b o}^{+}<r_{m s o}^{+}$. The second region, Region IIIb, with 

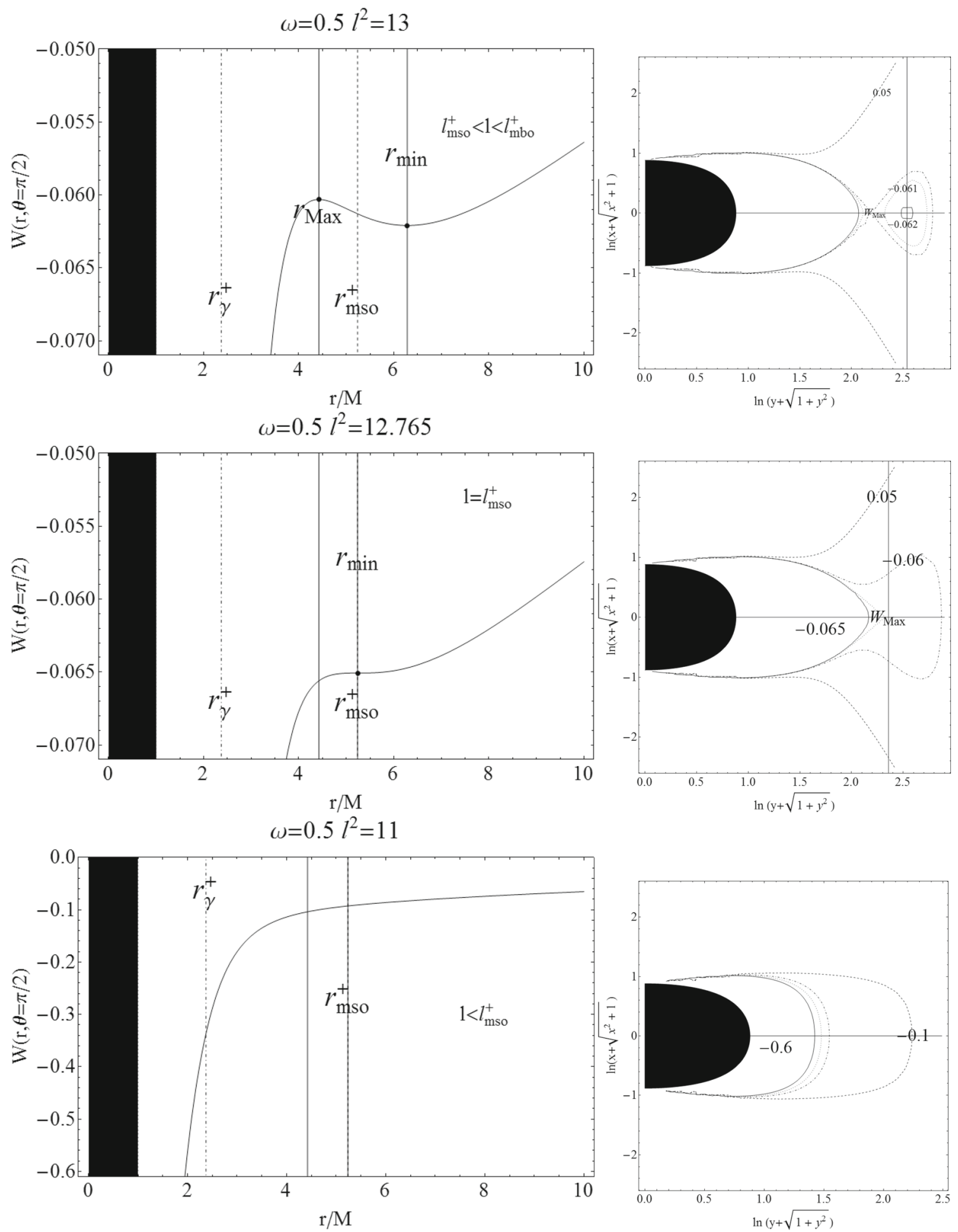

Fig. 19 Extreme black hole case: $\omega M^{2}=0.5$. We have $r_{ \pm}=$ $M$ with $r_{ \pm}<r_{\gamma}^{+}<r_{m b o}^{+}<r_{m s o}^{+}$and $l_{\gamma}^{+}>l_{m b o}^{+}>$ $l_{m s o}^{+} \cdot r / M=\sqrt{x^{2}+y^{2}}$ and $(x, y)$ are Cartesian coordinates. The

$\omega \in] \omega_{c}, \omega_{\gamma}[$, investigated in Sect. 6.1.7, is defined by the radii $r_{\text {stat }}<r_{m b o}^{-}<r_{\Omega}^{\mathrm{Max}}<r_{m s o}^{-}<r_{m b o}^{+}<r_{m s o}^{+}$. The Region IV with $\omega \in] \omega_{\gamma}, 0.5[$, considered in Sect. 6.1.9, black region is $r<r_{+}$. Here $\omega M^{2} \rightarrow \omega$. Vertical lines in theright panels set the $r_{i} \in \mathscr{R}$ and the effective potential critical points

contains two photon circular orbits, the inner stable one with $r_{\gamma}^{-}$, the outer unstable one at $r_{\gamma}^{+}$, and the marginally stable circular orbit $r_{m s o}^{+}$; we have $r_{\text {stat }}<r_{m b o}^{-}<r_{\gamma}^{-}<r_{\gamma}^{+}<r_{m b o}^{+}<$ 

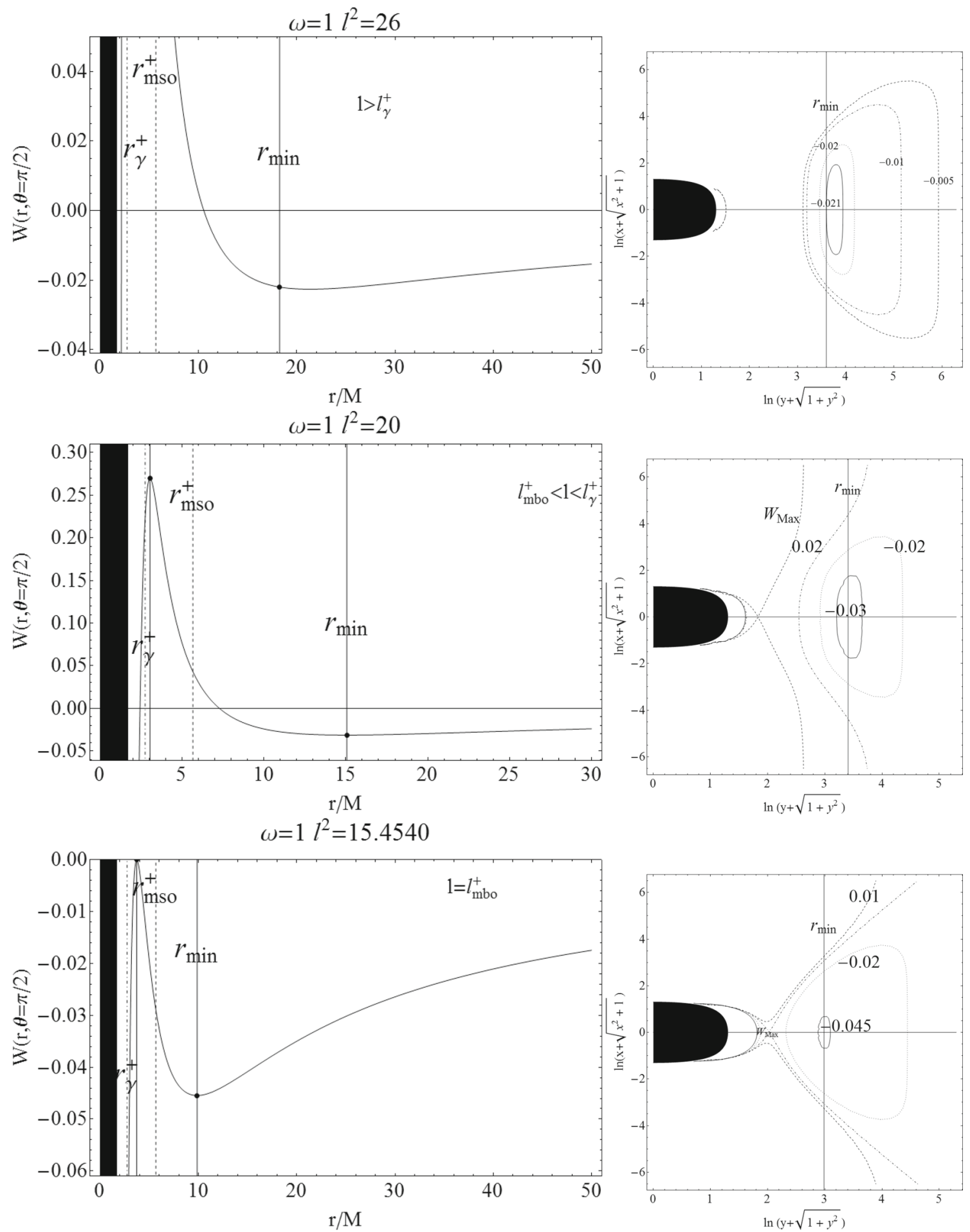

Fig. 20 Black hole case. Vertical lines in the right panels set the $r_{i} \in \mathscr{R}$ and the effective potential critical points. We have $\omega M^{2}=1$ with $r_{+}=(1+1 / \sqrt{2}) M$ with $r_{+}<r_{\gamma}^{+}<r_{m b o}^{+}<r_{m s o}^{+}$and

$l_{\gamma}^{+}>l_{m b o}^{+}>l_{m s o}^{+} . r / M=\sqrt{x^{2}+y^{2}}$ and $(x, y)$ are Cartesian coordinates. The black region is $r<r_{+}$. We have $\omega M^{2} \rightarrow \omega$

$r_{m s o}^{+}$. In the curve $\left.\Delta_{\gamma}^{ \pm} \equiv\right] r_{\gamma}^{-}, r_{\gamma}^{+}[$, no circular geodesics are allowed. The situation in the black hole case $\omega M^{2}>0.5$, detailed in Sect. 6.3, in the region $r>r_{+}$, is regulated by the existence of a photon orbit $r_{\gamma}>r_{+}$, a marginally bounded orbit $r_{m b o}>r_{\gamma}$, and a marginally stable orbit $r_{m s o}>r_{m b o}$.

The different properties of toroidal configurations in $\mathrm{K}-\mathrm{S}$ spacetimes entail the signatures of some K-S naked singu- 

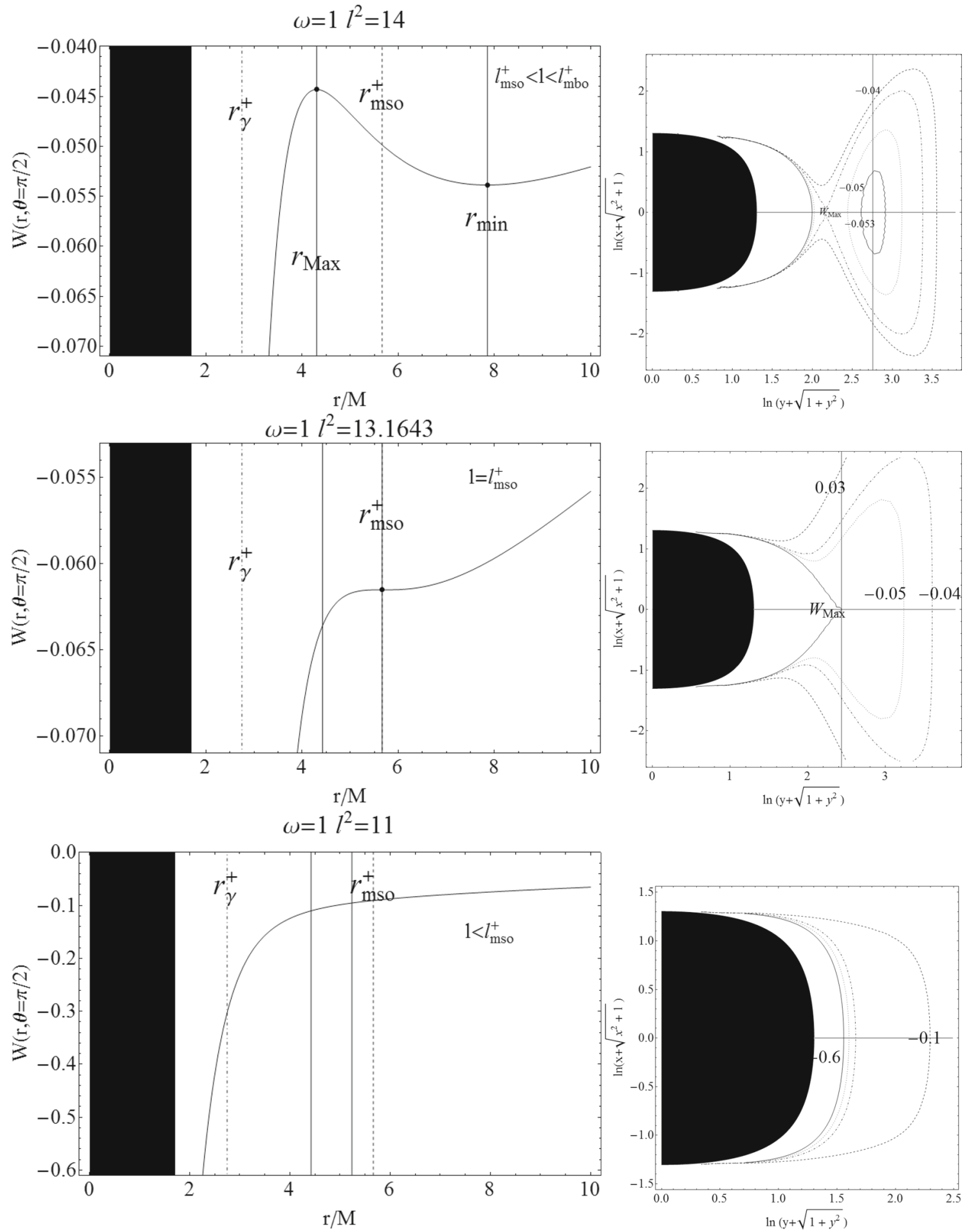

Fig. 21 Black hole case. We have $\omega M^{2}=1$ with $r_{+}=(1+$ $1 / \sqrt{2}) M, r_{+}<r_{\gamma}^{+}<r_{m b o}^{+}<r_{m s o}^{+}$and $l_{\gamma}^{+}>l_{m b o}^{+}>l_{m s o}^{+}$. $r / M=\sqrt{x^{2}+y^{2}}$ and $(x, y)$ are Cartesian coordinates. The black

larity spacetimes that enable us to clearly distinguish them from the black hole backgrounds due to the appearance of accretion phenomena. region is $r<r_{+}$. We have $\omega M^{2} \rightarrow \omega$. Vertical lines in the right panels set the $r_{i} \in \mathscr{R}$ and the effective potential critical points

We can summarize the main differences between the fluid configurations in $\mathrm{K}-\mathrm{S}$ naked singularity and black hole spacetimes as follows. 
For the spacetimes in the Regions II-III-IV there are two types of particle orbits and fluid configurations to be considered, associated to the outer and inner circular orbits $( \pm)$, far from and close to the attractor, respectively. This distinction makes the matter dynamics in these geometries very similar to the case of corotating and counterrotating particles orbiting in the Kerr geometry or electrically neutral particles in the case of charged spherically symmetric attractors. However, in the $\mathrm{K}-\mathrm{S}$ cases, as for the electrically neutral particles orbiting a Reissner-Nordström spacetime, this peculiar feature exclusively is due to the geometric properties of a naked singularity spacetimes when the Hořava parameter is $\omega \in] \omega_{m b o}, 0.5[$. A more general discussion of the analogies between the Kehagias-Sfetsos and the Reissner-Nordström solutions can be found in $[31,65]$.

Associated to the two sets of orbits in a naked singularity of Region $I V$ there are a couple of photon orbits, the inner stable one and the outer unstable one, defining a halo where no cusp or disc center can be located. In the black hole case only one limiting photon orbit exists. In naked singularity spacetimes of Regions $I I-I V$, there are a couple of stable orbits, and a couple of last bounded orbits in Regions III$I V$. The properties of test particle orbits in the BH and NS attractors are detailed and discussed in $[31,35]$.

In the spacetimes of Regions $I-I I I$ the relativistic angular velocity $\Omega$ has a critical point (as a function of $r / M$ ) located in $r_{\Omega}^{\mathrm{Max}}$. The exact location of this orbit with respect to the radii in the set $\mathscr{R}$ and in particular $r_{m b o}^{-}$, as $r_{m b o}^{-}\left(\omega_{c}\right)=$ $r_{\Omega}^{\mathrm{Max}}\left(\omega_{c}\right)$, makes it convenient to distinguish naked singularity sources in Region III from those with $\omega \in] \omega_{m b o}, \omega_{c}[$ (Region IIIa) and those with $\omega \in] \omega_{c}, \omega_{\gamma}[$ (Region IIIb). These cases are discussed Sects. 6.1.5 and 6.1.7, respectively, the main difference being in the location of $W_{\text {crit }}>0$, leading to the open or closed critical configurations.

In all the naked singularity spacetimes there is a "static limit" where $l_{\text {stat }}=0$. Generally stable orbits can be located in $r>r_{\text {stat }}$. The region $r>r_{\text {stat }}$ includes in geometries of Regions $I I-I V$ a disconnected, unstable orbital range in Regions II-IIIb and a forbidden halo in Region IV. The existence of a static limit could be interpreted as a repulsive gravity effect due to the naked singularity. We should note, however, that $r_{\text {stat }}$ decreases with $\omega M^{2}$, and as a consequence of this fact as $\omega$ approaches the value for the extreme black hole case, the regions where a (stable) matter configuration can be located closes the singularity, even if there cannot be a proper accretion point. This peculiar structure of the stability properties of a spacetime with a naked singularity can be compared with the analogous situation for the exact solutions of Einstein equations, for example in the Reissner-Nordström case [66,67], or the cosmological solutions of the Kerr-de Sitter or Reissner-Nordström-de Sitter cases [51,62]. However, at $r>r_{\text {stat }}$ there is always a curve of stable (closed) configurations.
The closed configurations in the black hole geometries are associated to an inner matter surface embracing the black hole; see for example Fig. 20. In the naked singularity spacetimes in general there are exterior open surfaces (as $W_{\text {Max }} \geq 0$ ) associated to the closed inner ones, whose distance is regulated by the gap between the critical points of the function $W$; see for example Fig. 9. The open equipotential surfaces could be relevant for the description of the outflow of matter in jets or winds.

Because of the presence of two minimum points for the fluid effective potential in the naked singularities of Regions $I I-I V$ it is possible to identify three types of configurations corresponding to the following three cases: $I W_{\min }^{-}>W_{\min }^{+}$, $I I W_{\min }^{-}=W_{\min }^{+}$, and finally $I I I W_{\min }^{-}<W_{\min }^{+}$. Their properties are discussed in Sect. 6.1.3. The alternation of these configurations characterizes and distinguishes the different cases from the Region II. Since $W_{\min }^{+}<0$, the configuration is always of kind $I$ when $W_{\min }^{-}>0$, and a typical profile is shown in Fig. 13. In this case, $W_{\text {Max }}>0$ corresponds to excretion crossed configurations opened toward the outer regions. These discs occur in the naked singularity spacetimes of Regions $I I I-I V$ where the radii $r_{m b o}^{ \pm}$are defined. Then it could be possible that $W_{\min }^{-}<0$, and it may be so that $W_{\text {Max }}>0$ as well; see for example Fig. 17c, the case $W_{\text {Max }}=0$, or $W_{\text {Max }}>0$ in Fig. 6. However, the sign of the maximum values only determines the open or closed topology of the unstable configurations.

Focusing on the minimum values of $W$ : it can be of kind $I, W_{\min }^{-}>W_{\min }^{+}$, see for example Fig. 8, or $W_{\min }^{-}<W_{\min }^{+}$, see for example Fig. 6. The second, limiting case, is $I I-W_{\min }^{-}=W_{\text {Max }}^{+}$, which satisfies the conditions: $W\left(r_{\min }^{-}, l\right)=W\left(r_{\min }^{+}, l\right), \partial_{r} W\left(r_{\min }^{-}, l\right)=\partial_{r} W\left(r_{\min }^{+}, l\right)=0$, and $\partial_{r}^{2} W\left(r_{\min }^{-}, l\right)>0, \partial_{r}^{2} W\left(r_{\min }^{+}, l\right)>0$.

In the geometries of Regions II-IV, configurations can lead to the excretion of matter toward the outer space but not accretion, see also $[51,62]$ for similar cases. Correlated to this, for $l=l_{m s o}^{-}$, there is an inner cusp of the potential, in $r_{m s o}^{-}$, and an outer cusp in $r_{m s o}^{+}$with $l=l_{m s o}^{+}$. Consequently there are a couple of stable (or crossed) closed configurations at equal $l$ and $K$, generating an inner and outer disc (corresponding to two minima for the Keplerian disc effective potential). The two sets of closed configurations can be separated by an open crossed configuration with an outer cusp (as only excretion is possible, not accretion), when $W_{\text {Max }} \geq 0$, or by a closed crossed surface with two centers in the maxima points of the pressure, when $W_{\text {Max }}<0$. In such a binary system feeding of matter can occur from one configuration (the inner smaller one) to the companion (the outer larger one). It is possible for $W \in] W_{\text {Max }}, 0$ [ that one closed configuration occurs with two maxima of the thickness for the two extrema of the pressure $p_{\mathrm{Max}}$ for the outer and inner configurations, respectively. These double "centers" are clearly related to the three classes of configurations introduced in Region III. 
We can conclude that the character of the toroidal equilibrium configurations of perfect fluid could give clear signatures of the presence of the Kehagias-Sfetsos naked singularities representing a simple solution of the laws of the Hořava quantum gravity.

Concerning the classification of the tori, we can separate classes where only the non-accreting equilibrium structures are possible in the spacetimes with $\omega<\omega_{m s o}$, and the classes of standard single accreting toroidal structures, or the doubled structures allowing for accretion, or forbidding the accretion but allowing for feeding from the outer torus to the inner torus, or with outflow to the outer space that can occur in the K-S naked singularity spacetimes with $\omega>\omega_{m s o}$. In the $\mathrm{K}-\mathrm{S}$ black hole spacetimes $(\omega>1 / 2)$, the classification of the toroidal configurations is similar to the standard Schwarzschild spacetime.

Observational signatures of the K-S naked singularities spacetimes can be obtained due to modeling of the optical phenomena (continuum spectrum, profiled spectral lines) related to these compact objects [30], as has been done for the case of the thin, Keplerian accretion disks [35]. We have shown that the toroidal structures in the $\mathrm{K}-\mathrm{S}$ naked singularity spacetimes demonstrate two most profound signatures. First, the existence of doubled toroidal configurations having the same $l=$ const that occurs in the spacetimes with $\omega>\omega_{m s o}$ (NS of Regions II-IV). In some situations the outer toroid can feed the inner one, while in others the feeding is forbidden, but outflow of matter into the outer space is allowed across the cusp of the outer toroid (in geometries of Regions II-IV, the configurations can lead to excretion of matter toward the outer space but not accretion, while the value of the double structures effective potential at the minimum points identifies and distinguishes cases $I-I I-I I I$ in the NS spacetimes with $\omega>\omega_{m s o}$ ). Second, tori with a cusp cannot exist in the spacetimes with $\omega<\omega_{m s o}$, as unstable circular geodesics cannot exist in such spacetimes. Accretion due to the Paczynski mechanism of mechanical balance violation is not possible in these $\mathrm{K}-\mathrm{S}$ naked singularity spacetimes, and mass could be strongly accumulated in such tori, leading possibly to the creation of toroidal stars, if the nuclear reactions will be started at these structures. The mass outflows are possible due to violation of the perfect fluid assumption for behavior of the matter in the tori. However, we stress that the standard Keplerian accretion discs are limited by the condition $\mathrm{d} \Omega_{K} / \mathrm{d} r<0$, required by the Magneto-Rotational Instability (MRI) viscosity mechanism. In fact, if MRI is the only mechanism generating the viscosity effects at the basis of the accretion of matter in these disks, then it follows that the disk inner edge has to be located at the critical radius $r_{\Omega}^{\text {Max }}$. The gradient of the angular frequency (for the circular geodesic motion) vanishes at $r_{\Omega}^{\mathrm{Max}}$ inside the Keplerian disc in all the $\mathrm{K}-\mathrm{S}$ naked singularity spacetimes. Therefore the condition for Keplerian accretion is violated there. As for the $\mathrm{K}-\mathrm{S}$ naked singularity spacetimes of Region I this would imply the limit from below of the fluid angular momentum considering $l_{\Omega}^{\mathrm{Max}} \equiv l\left(r_{\Omega}^{\mathrm{Max}}\right)>l_{\text {stat }}$, where $\Omega_{K}\left(r_{\text {stat }}\right)=0$, and we expect that, near the radius $r_{\Omega}^{\mathrm{Max}}=4^{1 / 3} r_{\text {stat }}$, matter is accumulating.

Acknowledgments The authors acknowledge the Institutional support of the Faculty of Philosophy and Science of the Silesian University at Opava. ZS and JS acknowledge the Albert Einstein Centre for gravitation and astrophysics supported by the Czech Science Foundation Grant No. 14-37086G. ZS, JS and HK acknowledge the internal student grant of the Silesian University SGS/23/2013.

Open Access This article is distributed under the terms of the Creative Commons Attribution 4.0 International License (http://creativecomm ons.org/licenses/by/4.0/), which permits unrestricted use, distribution, and reproduction in any medium, provided you give appropriate credit to the original author(s) and the source, provide a link to the Creative Commons license, and indicate if changes were made.

Funded by SCOAP . $^{3}$.

\section{References}

1. P. Hořava, Phys. Rev. D 79, 8 (2009)

2. E.M. Lifshitz, Zhurnal Esperimentalnoy i Teoretiskoy Fyziki 255, $269(1941)$

3. P. Hořava, Phys. Rev. Lett. 102, 16 (2009)

4. M. Henneaux, A. Kleinschmidt, G. Lucena Gómez, Phys. Rev. D 81, $064002(2010)$

5. P. Hořava, ChM Melby-Thompson, Phys. Rev. D 82, 064027 (2010)

6. Ch. Anderson, S.J. Carlip, J.H. Cooperman, P. Hořava, R.K. Kommu, P.R. Zulkowski, Phys. Rev. D 85, 044027 (2012)

7. T. Griffin, P. Hořava. Ch.M. Melby-Thompson, J. High Energy Phys 2012(5), 10 (2012). doi:10.1007/JHEP05(2012)010

8. T. Griffin, P. Hořava, ChM Melby-Thompson, Phys. Rev. Lett. 110, 081602 (2013)

9. D. Vernieri, T.P. Sotiriou, Phys. Rev. D 85, 069901 (2012)

10. S. Liberati, L. Maccione, T.P. Sotiriou, Phys. Rev. Lett. 109, 151602 (2012)

11. E. Barausse, T.P. Sotiriou, Phys. Rev. Lett. 109, 181101 (2012)

12. E. Barausse, T.P. Sotiriou, Phys. Rev. Lett. 110, 039902 (2013)

13. E. Barausse, T.P. Sotiriou, Phys. Rev. D 87, 087504 (2013)

14. A. Kehagias, K. Sfetsos, Phys. Lett. B 678, 123-126 (2009)

15. M.-I. Park, J. High Energy Phys. 9, 123 (2009)

16. E.B. Kiritsis, G. Kofinas, JHEP 1001, $122(2010)$

17. L. Iorio, M.L. Ruggiero, Int. J. Mod. Phys. A 25, 5399-5408 (2010)

18. M. Liu, J. Lu, B. Yu, J. Lu, Gen. Relativ. Gravit. 43, 1401-1415 (2011)

19. L. Iorio, M.L. Ruggiero, Int. J. Mod. Phys. D 20, 1079-1093 (2011)

20. A. Hakimov, B. Turimov, A. Abdujabbarov, B. Ahmedov, Mod. Phys. Lett. A 25, 3115-3127 (2010)

21. A.N. Aliev, Ç. Şentürk, Phys. Rev. D 82, 104016 (2010)

22. A. Abdujabbarov, B. Ahmedov, A. Hakimov, Phys. Rev. D 83, 044053 (2011)

23. V. Enolskii, B. Hartmann, V. Kagramanova, J. Kunz, C. LÃd'mmerzahl, P. Sirimachan, Phys. Rev. D 84, 084011 (2011)

24. Z. Horváth, L.Á. Gergely, Z. Keresztes, T. Harko, F.S.N. Lobo, Phys. Rev. D 84, 083006 (2011)

25. A. Hakimov, A. Abdujabbarov, B. Ahmedov, Phys. Rev. D 88, 024008 (2013)

26. T. Harko, Z. Kovács, F.S.N. Lobo, Class. Q. Grav. 28, 165001 (2011) 
27. L. Amarilla, E.F. Eiroa, Phys. Rev. D 85, 064019 (2012)

28. E.F. Eiroa, C.M. Sendra, Phys. Rev. D 86, 083009 (2012)

29. F. Atamurotov, A. Abdujabbarov, B. Ahmedov, Astrophys. Space Sci. 348, 179 (2013)

30. F.S. Guzmán, J.M. Rueda-Becerril, Phys. Rev. D 80(8), 084023 (2010). doi:10.1103/PhysRevD.80.084023

31. R.S.S. Vieira, J. Schee, W. Kluźniak, Z. Stuchlík, M. Abramowicz, Phys. Rev. D 90, 024035 (2014)

32. Z. Stuchlík, S. Hledík, Acta Phys. Slov. 52, 363 (2002)

33. D. Pugliese, H. Quevedo, R. Ruffini, Phys. Rev. D 83, 024021 (2011)

34. Z. Stuchlík, J. Schee, A. Abdujabbarov, Phys. Rev. D 89, 104048 (2014)

35. Z. Stuchlik, J. Schee, Class. Q. Grav. 31, 195013 (2014)

36. A. Kotrlová, Z. Stuchlík, G. Török, Class. Q. Grav. 25, 225016 (2008)

37. Z. Stuchlík, A. Kotrlová, Gen. Relativ. Gravit. 41, 1305 (2009)

38. J. Schee, Z. Stuchlík, Int. J. Mod. Phys. D 18, 983-1024 (2009)

39. Z. Stuchlík, M. Kološ, J. Cosmol. Astropart. Phys. 10, 008 (2012)

40. A.N. Aliev, G. Daylan Esmer, P. Talazan, Class. Q. Grav. 30, 045010 (2013)

41. Z. Stuchlík, Bull. Astron. Inst. Czechoslov. 31, 129-144 (1980)

42. Z. Stuchlík, J. Schee, Class. Q. Grav. 27, 215017 (2010)

43. Z. Stuchlík, S. Hledík, K. Truparová, Class. Q. Grav. 28, 155017 (2011)

44. M. Patil, P. Joshi, Class. Q. Grav. 28, 23 (2011)

45. Z. Stuchlík, J. Schee, Class. Q. Grav. 29, 065002 (2012)

46. Z. Stuchlík, J. Schee, Class. Q. Grav. 30, 075012 (2013)

47. J. Schee, Z. Stuchlík, J. Cosmol. Astropar. Phys. 4, 005 (2013)

48. M. Kološ, Z. Stuchlík, Phys. Rev. D 88, 065004 (2013)

49. K. Adámek, Z. Stuchlík, Class. Q. Grav. 30, 205007 (2013)

50. D. Capasso, A.P. Polychronakos, J. High Energy Phys. 2, 68 (2010)

51. H. Kučáková, P. Slaný, Z. Stuchlík, J. Cosmol. Astropart. Phys. 1, 033 (2011)

52. V.V. Kiselev, Class. Q. Grav. 20, 1187 (2003)

53. L. Iorio, M.L. Ruggiero, Open Astron. J. 3, 167 (2010)
54. M. Dwornik, Z. Horváth, L.Á. Gergely, Astron. Nachr. 334, 1039$1042(2013)$

55. J. Chen, Y. Wang, Int. J. Mod. Phys. A 25, 1439 (2010)

56. Z. Stuchlík, J. Schee, Class. Q. Grav. 29, 025008 (2012)

57. S.A. Balbus, J.F. Hawley, Rev. Mod. Phys. 70, 1-53 (1998)

58. M. Kozlowski, M. Jaroszynski, M.A. Abramowicz, Astron. Astrophys. 63, 209-220 (1978)

59. Z. Stuchlík, P. Slaný, S. Hledík, Astron. Astrophys. 363, 425-439 (2000)

60. Z. Stuchlík, Mod. Phys. Lett. A 20, 561-575 (2005)

61. M.A. Abramowicz, M. Calvani, L. Nobili, Astrophys. J. 242, 772788 (1980). doi:10.1086/158512

62. P. Slaný, Z. Stuchlík, Class. Q. Grav. 22, 3623-3651 (2005)

63. Z. Stuchlík, P. Slaný, J. Kováŕ, Class. Q. Grav. 26, 215013 (2009)

64. D. Pugliese, G. Montani, M.G. Bernardini, Mon. Not. R. Astron. Soc. 428(2), 952 (2012)

65. W. Janke, D.A. Johnston, R. Kenna, J. Phys. A 43, 425206 (2010)

66. D. Pugliese, H. Quevedo, R. Ruffini, Phys. Rev. D 83, 024021 (2011). arXiv:1303.6250 [gr-qc]

67. D. Pugliese, H. Quevedo, R. Ruffini, Phys. Rev. D 88(2), 024042 (2013)

68. F.D. Lora-Clavijo, P.A. Ospina-Henao, J.F. Pedraza, Phys. Rev. D 82, 084005 (2010)

69. Y.S. Myung, Phys. Lett. B 685, 318 (2010)

70. Y.S. Myung, Phys. Lett. B 684, 318 (2010)

71. M. Liu, J. Lu, Phys. Lett. B 699(4), 296-300 (2011)

72. I. Radinschi, F. Rahaman, A. Banerjee, Int. J. Theor. Phys. 50, 2906 (2011)

73. M. Eune, B. Gwak, W. Kim, Phys. Lett. B 718, 1505 (2013)

74. H.W. Lee, Y.-W. Kim, Y.S. Myunga, Eur. Phys. J. C 70(1), 367-371 (2010)

75. J. Suresh, V.C. Kuriakose, Gen. Relativ. Gravit. 45, 2013 (1877)

76. P. Slany, J. Kovar, Z. Stuchlik, V. Karas, Astrophys. J. Suppl. 205, 3 (2013)

77. C. Cremaschini, J. Kovár, P. Slaný, Z. Stuchlík, V. Karas, Astrophys. J. Suppl. 209, 15 (2013). arXiv:1309.3979 [astro-ph.HE] 